\title{
Radio-Astronomical Imaging in the Presence of Strong Radio Interference
}

\author{
Amir Leshem, Member, IEEE, and Alle-Jan van der Veen, Member, IEEE
}

\begin{abstract}
Radio-astronomical observations are increasingly contaminated by interference, and suppression techniques become essential. A powerful candidate for interference mitigation is adaptive spatial filtering. We study the effect of spatial filtering techniques on radio-astronomical imaging. Current deconvolution procedures, such as CLEAN, are shown to be unsuitable for spatially filtered data, and the necessary corrections are derived. To that end, we reformulate the imaging (deconvolution/calibration) process as a sequential estimation of the locations of astronomical sources. This not only leads to an extended CLEAN algorithm, but also the formulation allows the insertion of other array signal processing techniques for direction finding and gives estimates of the expected image quality and the amount of interference suppression that can be achieved. Finally, a maximum-likelihood (ML) procedure for the imaging is derived, and an approximate ML image formation technique is proposed to overcome the computational burden involved. Some of the effects of the new algorithms are shown in simulated images.
\end{abstract}

Index Terms-CLEAN, interference mitigation, maximum likelihood, minimum variance, parametric imaging, radio astronomy, spatial filtering, synthesis imaging.

\section{INTRODUCTION}

$\mathbf{T}$ HE future of radio-astronomical discoveries depends on achieving better resolution and sensitivity while maintaining immunity to terrestrial interference. The last two demands are obviously contradictory as improved sensitivity implies receiving more interfering signals. One possible track is to switch to massive phased array technology. If instead of the huge dishes which became the trademark of radio astronomy, we use phased-array radio telescopes comprised of tens of thousands of small elements, then we gain both in terms of resolution and sensitivity while increasing the flexibility to mitigate interference. The international effort in this direction is coordinated under the framework of the Square Kilometer Array project (SKA). In this paper, we try to analyze the effect of on-line interference rejection on the image formation process in such an instrument.

We briefly describe the current status of radio-astronomical imaging; for a more extensive overview the reader is referred to [39], [44], or [26]. The principle of radio interferometry has

\footnotetext{
Manuscript received September 2, 1999; revised March 21, 2000. The work of A. Leshem was supported by the NOEMI project of the STW under Contract DEL77-4476. The material in this paper was presented in part at the workshop "Perspectives in Radio-Astronomy: Technologies for Large Arrays," Dwingeloo, The Netherlands 1999.

The authors are with the Deptartment of Electrical Engineering, Delft University of Technology 2628 CD Delft, The Netherlands (e-mail: leshem@cas.et.tudelft.nl; allejan@ cas.et.tudelft.nl).

Communicated by J. A. O'Sullivan, Guest Editor.

Publisher Item Identifier S 0018-9448(00)06072-7.
}

been used since 1946 when Ryle and Vonberg constructed a radio interferometer using dipole antenna arrays [29]. During the 1950's several radio interferometers, which use synthetic apertures created by movable antennas, have been constructed. In 1962, the principle of aperture synthesis using Earth rotation were proposed [30]. The basic idea is to exploit the rotation of the Earth to obtain denser coverage of the visibility domain (spatial Fourier domain).

The first instrument to use this principle was the 5-km Cambridge radio telescope. During the 1970's, new instruments with large apertures were constructed. Among these are the Westerbork Synthesis Radio Telescope (WSRT) in the Netherlands and the Very Large Array (VLA) in the USA. Even these instruments subsample the Fourier domain, so that unique reconstruction is not possible without some further processing known as deconvolution. The deconvolution process uses some a priori knowledge about the image to remove the effect of "dirty beam" sidelobes.

Two principles dominate astronomical imaging deconvolution. The first method was proposed by Hogbom [12] and is known as CLEAN. The CLEAN method is basically a sequential Least Squares (LS) fitting procedure in which the brightest source location and power are estimated. The response of this source is removed from the image, then the process continues to find the next brightest source, and so on, until the residual image is noise-like. During the years, it has been partially analyzed [31], [32], and [37]. However, full analysis of the method is still lacking due to its iterative nature.

A second method proposed by Jaynes [13], [14] is maximumentropy deconvolution (MEM). The basic idea behind MEM is the following. Among all images which are consistent with the measured data and the noise distribution not all satisfy the positivity demand, i.e., the sky brightness is a positive function. Consider only those that satisfy the positivity demand. From these select the one that is most likely to have been created randomly. This idea has also been proposed in [8] and applied to radio-astronomical imaging in [11]. Other approaches based on the differential entropy have also been proposed [1] and [43]. An extensive collection of papers discussing the various methods and aspects of maximum entropy can be found in the various papers in [27].

The above-mentioned algorithms assume perfect knowledge of the instrumental response (point-spread function). Due to various internal and external effects this assumption holds only approximately. One way to overcome this problem is the use of calibrating sources. An unresolved source with known parameters is measured, and by relating the model errors to the array elements a set of calibration equations is solved. A much more 
appealing solution is to try to improve the fitting between the data and the sky model by adjusting the calibration parameters. Another possibility [23] is to use the redundant structure of the array to solve for the calibration parameters (this is possible only for some arrays which have redundant baselines, such as the WSRT). A good overview of the various techniques is given in [25].

A major problem facing radio astronomy is the accelerated use of the electromagnetic spectrum. Even in bands which are reserved to radio-astronomical observation one can find interference, e.g., sidelobes of emissions from the Iridium or GLONASS satellites. As was shown in [38], interferometric arrays are less sensitive to interference than single-dish instruments. However, the interference still appears in images, especially for observations at frequency bands not specifically reserved for radio astronomy. Many efforts all over the world are currently put into improving the interference mitigation capabilities of radio telescopes. The methods considered span over all possible domains: temporal [42], [10], [3], spatiotemporal [15], spatiospectral [21], [18], [17], and wavelet-based methods [22]. Also considered are techniques which use statistical and deterministic properties such as non-Gaussianity and constant modulus of the interferers, in order to use blind beamforming to remove the interferers.

As far as multielement synthesis imaging radio telescopes are concerned, the spatial methods are extremely appealing, since each interferer has its own "spatial signature." Estimating these signatures enables efficient mitigation of the interferer using phased-array techniques. However, important questions arise. As we will show in this paper, applying time-varying spatial filters makes the point-spread function space-varying. In particular, the image formation techniques will have to be modified accordingly. To cope with this problem, we reformulate the classical Fourier imaging framework in a parametric manner more appropriate for statistical analysis of the interference mitigation. This enables us to incorporate the spatial filtering naturally into the image formation process.

Our reformulation of the image formation problem describes the measurements as a set of covariance matrices, measured at the various observation epochs. This yields a model where the array response is time-varying. Previous research on time-varying arrays and their application to direction-of-arrival (DOA) estimation includes [45], where an ML estimator for a single source and the corresponding Cramer-Rao bound (CRB) are derived. For multiple sources [9] proposes three approaches: a modified beamforming, a virtual interpolated array, and focusing matrices. The main drawback of the last two methods is that they do not lend themselves to estimating more sources than the number of physical sensors. In [34], a generalized LS (GLS) approach is proposed. The idea is to present the problem of estimating the source powers (given the DOA parameters) as a linear problem. This enables a closed-form solution for the powers. Substituting back into the GLS estimator, the problem is reduced to a multidimensional search problem over the DOA's. The main drawback of this approach is the need to invert very large matrices $\left(p^{2} \times p^{2}\right.$, where $p$ is the number of physical sensors). It is also shown that the asymptotic performances of the GLS and the ML estimators are identical up to second order, which makes it very attractive. All these papers deal with the case of a fully known array response and are limited to one-dimensional location parameters. In this paper, we also extend the above methods in several directions. First, we use the eigenstructure to mitigate strong interferers for which we do not know the array response, and we analyze the efficiency of this procedure. Second, we propose other relatively "low" complexity approaches, based on eigenstructure and Minimum Variance Distortionless Response (MVDR). Finally, we discuss an ML approach to the astronomical image formation problem, derive an approximate ML estimator (AML) of low complexity and discuss the relation of the AML to the CLEAN algorithm. Our treatment of the radio-astronomical imaging can be applied to other problems of imaging with time-varying sensor responses, such as ISAR/SAR radar imaging in the presence of strong jammers. The model then generalizes the model given in [35] to the time-varying context. For the case of an array with constant time behavior (connected element array), methods of computing the MLE have been proposed in [35] using the EM method or [7] using the SAGE algorithm. However, there is no straightforward extension of these methods to our context. Although an EM algorithm can be applied, the dimensionality of the parameter space makes it infeasible to perform full MLE without very good initialization. Reference [24] contains an extensive overview on image formation principles and algorithms, as well as previous work on parametric image formation in other fields such as radar and tomography.

To allow the paper to be of use both to the information theory/signal processing and to the radio astronomical communities the introductory part is of a tutorial nature. The structure of the paper is as follows. In Section II we describe the astronomical measurement process and introduce an often-used coordinate system. The measurement equation is subsequently rephrased in a more convenient matrix formulation in Section III, and extended with the effect of interference. In Section IV, we describe several basic spatial filtering approaches to on-line interference suppression, and compute the residual interference after adaptive estimation of its parameters for one specific case. In Sections V and VI, we discuss the image formation process, first based on classical techniques (CLEAN), then extended to other beamforming methods and taking the spatial filtering into account. Finally, we derive an approximate ML algorithm for image formation. We end with conclusions regarding future implementation of on-line interference suppression in radio astronomy.

\section{AStronomical MEASUREMENT EQUATIONS}

In this section we describe a simplified mathematical model for the astronomical measurement and imaging process. Our discussion follows the introduction in [26]. We begin with the measurement equation, and reformulate it into a matrix form in the next section. This will allow us to obtain a uniform description of various astronomical imaging operations, such as deconvolution and self-calibration.

The signals received from the celestial sphere may be considered as spatially incoherent, wideband random noise. It is 


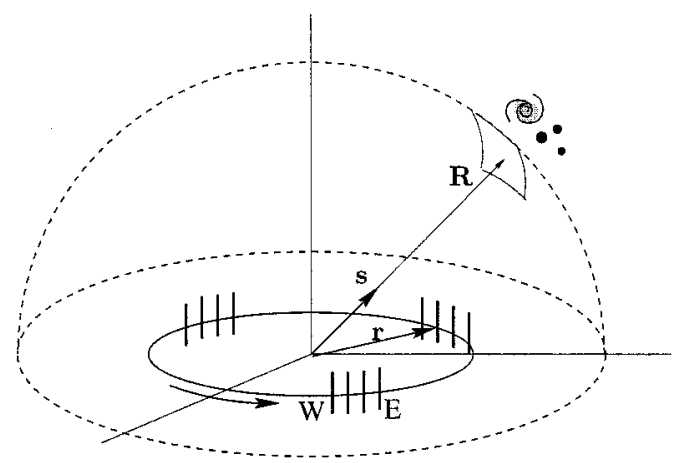

(a)

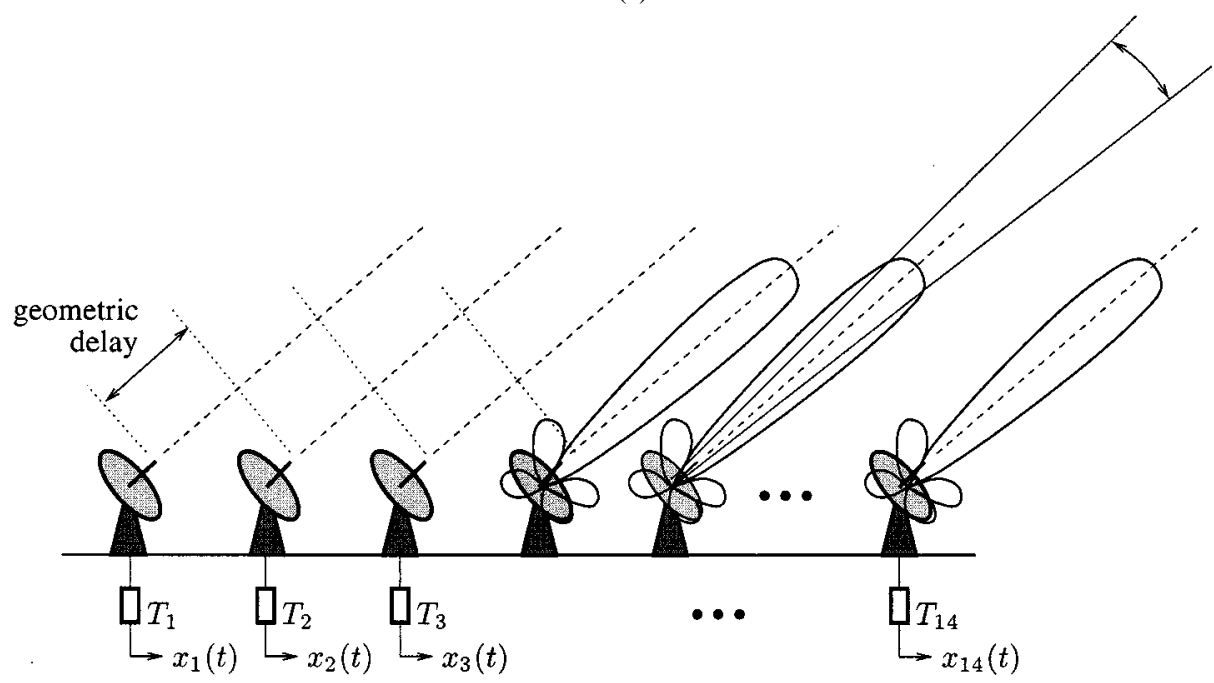

(b)

Fig. 1. (a) The emitted electrical field from the celestial sphere is received by a rotating telescope array. (b) Geometrical delay compensation.

possibly polarized and perhaps contains spectral absorption or emission lines. Rather than considering the emitted electric field at a location on the celestial sphere, astronomers try to recover the intensity (or brightness) $I_{f}(\boldsymbol{s})$ in the direction of unit-length vectors $\boldsymbol{s}$, where $f$ is a specific frequency. Let $E_{f}(\boldsymbol{r})$ be the received celestial electric field at a location $\boldsymbol{r}$ on Earth (see Fig. 1(a)). The measured correlation of the electric fields between two identical sensors $i$ and $j$ with locations $\boldsymbol{r}_{i}$ and $\boldsymbol{r}_{j}$ is called a visibility and is (approximately) given by [26] ${ }^{1}$

$$
\begin{aligned}
V_{f}\left(\boldsymbol{r}_{i}, \boldsymbol{r}_{j}\right) & :=\mathrm{E}\left\{E_{f}\left(\boldsymbol{r}_{i}\right) \overline{E_{f}\left(\boldsymbol{r}_{j}\right)}\right\} \\
& =\int_{\mathrm{sky}} I_{f}(\boldsymbol{s}) e^{-2 \pi j f \boldsymbol{s}^{T}\left(\boldsymbol{r}_{i}-\boldsymbol{r}_{j}\right) / c} d \Omega .
\end{aligned}
$$

$\mathrm{E}\{\cdot\}$ is the mathematical expectation operator, the superscript $T$ denotes the transpose of a vector, and overbar denotes the complex conjugate. Note that $V_{f}$ is only dependent on the oriented distance $\boldsymbol{r}_{i}-\boldsymbol{r}_{j}$ between the two antennas; this vector is called a baseline.

For simplification, we may sometimes assume that the astronomical sky is a collection of $d$ discrete point sources (maybe

${ }^{1}$ To simplify notation, we do not include in our model the directional response of the elements of the radio telescope. This can be included in a straightforward manner as in [26, Ch. 1, Sec. 4.3] unresolved). This gives

$$
I_{f}(\boldsymbol{s})=\sum_{l=1}^{d} I_{f}\left(\boldsymbol{s}_{l}\right) \delta\left(\boldsymbol{s}-\boldsymbol{s}_{l}\right)
$$

where $\boldsymbol{s}_{l}$ is the coordinate of the $l$ th source, and then

$$
V_{f}\left(\boldsymbol{r}_{i}, \boldsymbol{r}_{j}\right)=\gamma \sum_{l=1}^{d} I_{f}\left(\boldsymbol{s}_{l}\right) e^{-2 \pi j f \boldsymbol{s}_{l}^{T}\left(\boldsymbol{r}_{i}-\boldsymbol{r}_{j}\right) / c} .
$$

Up to this point we have worked in an arbitrary coordinate system. For Earth rotation synthesis arrays, a coordinate system is often introduced as follows. We assume an array with antennas that have a small field of view and that track a reference source direction $\boldsymbol{s}_{0}$ in the sky. Other locations in the field of view can be written as $\boldsymbol{s}=\boldsymbol{s}_{0}+\boldsymbol{\sigma}, \boldsymbol{s}_{0} \perp \boldsymbol{\sigma}$ (valid for small $\boldsymbol{\sigma}$ ) and a natural coordinate system is

$$
\boldsymbol{s}_{0}=[0,0,1]^{T} \quad \sigma=[\ell, m, 0]^{T} .
$$

Similarly, for a planar array, the receiver baselines can be parameterized as

$$
\boldsymbol{r}_{i}-\boldsymbol{r}_{j}=\lambda[u, v, w]^{T}, \quad \lambda=\frac{c}{f} .
$$


The measurement equation in $(u, v, w)$ coordinates thus becomes

$$
V_{f}(u, v, w)=e^{-2 \pi j w} \iint I_{f}(\ell, m) e^{-2 \pi \jmath(u \ell+v m)} d \ell d m .
$$

The factor $e^{-2 \pi j w}$ is caused by the geometrical delay associated to the reference location, and can be compensated by introducing a slowly time-variant delay (see Fig. 1(b)). This synchronizes the center of the field-of-view and makes the reference source location appear as if it were at the North Pole. After compensation, we arrive at a measurement equation in $(u, v)$ coordinates only

$$
V_{f}(u, v)=\iint I_{f}(\ell, m) e^{-2 \pi \jmath(u \ell+v m)} d \ell d m .
$$

It has the form of a Fourier transform.

The function $V_{f}(u, v)$ is sampled at various coordinates $(u, v)$ by first taking all possible sensor pairs $i, j$ or baselines $\boldsymbol{r}_{i}-\boldsymbol{r}_{j}$, and second by realizing that the sensor locations $\boldsymbol{r}_{i}$, $\boldsymbol{r}_{j}$ are actually time-varying since the Earth rotates. Given a sufficient number of samples in the $(u, v)$ domain, the relation can be inverted to obtain an image (the "map"), which is the topic of Section V.

\section{ARRAY SignAl Processing Formulation}

\section{A. Obtaining the Measurements}

We will now describe the situation from an array signal processing point of view. The signals received by the antennas are amplified and down-converted to baseband. A time-varying delay for every antenna is also introduced, to compensate for the geometrical delay. Following traditional array signal processing practice, the signals at this point are called $x_{i}(t)$ rather than $E_{f}(\boldsymbol{r})$, and are stacked in vectors

$$
\boldsymbol{x}(t)=\left[x_{1}(t), \cdots, x_{p}(t)\right]^{T}
$$

where $p$ is the number of antennas. These are then processed by a correlation stage.

It will be convenient to assume that $\boldsymbol{x}(t)$ is first split by a bank of narrowband subband filters into a collection of frequency components $\boldsymbol{x}_{f}(t)$. The main output of the telescope hardware is then a sequence of empirical correlation matrices $\hat{\boldsymbol{R}}_{f}(t)$ of crosscorrelations of $x_{f}(t)$, for a set of frequencies $f \in\left\{f_{k}\right\}$ covering a $10-\mathrm{MHz}$ band or so, and for a set of times $t \in\left\{t_{k}\right\}$ covering up to 12 h. ${ }^{2}$ Each correlation matrix $\hat{\boldsymbol{R}}_{f}(t)$ is an estimate of the true covariance matrix $\boldsymbol{R}_{f}(t)$

$$
\begin{aligned}
\boldsymbol{R}_{f}(t) & =\mathrm{E}\left\{\boldsymbol{x}_{f}(t) \boldsymbol{x}_{f}(t)^{\mathrm{H}}\right\} \\
\hat{\boldsymbol{R}}_{f}(t) & =\frac{1}{N} \sum_{n=0}^{N-1} \boldsymbol{x}_{f}(t+n T) \boldsymbol{x}_{f}(t+n T)^{\mathrm{H}}
\end{aligned}
$$

where the superscript ${ }^{\mathrm{H}}$ denotes a complex conjugate transpose, $T$ is the sample period of $\boldsymbol{x}_{f}(t)$, and $N$ is the number of samples over which is averaged. The matrices $\hat{\boldsymbol{R}}_{f}(t)$ are stored for off-line spectral analysis and imaging. Typically, each subband

\footnotetext{
${ }^{2}$ Many telescope sites including WSRT follow actually a different scheme wherein the signals are first correlated at several lags and subsequently Fouriertransformed. This leads to similar results.
}

has a bandwidth on the order of $100 \mathrm{kHz}$ or less. Due to the subband filtering, the original sampling rate of $\boldsymbol{x}(t)$ is reduced accordingly, resulting in $T$ on the order of $10 \mu \mathrm{s}$ and the number of samples $N$ in the order of $10^{5}$ for each subband. $f$ represents the center frequency in a subband. From now on we consider the subbands independently ignoring that they are really connected. Consequently, in future equations we drop the dependence on $f$ in the notation.

The connection of the correlation matrices $R(t)$ to the visibilities $V(u, v)$ in Section II is as follows. Each entry $r_{i j}(t)$ of the matrix $\boldsymbol{R}(t)$ is a sample of this visibility function for a specific coordinate $(u, v)$ corresponding to the baseline vector

$$
\boldsymbol{r}_{i}(t)-\boldsymbol{r}_{j}(t)=\lambda\left[u_{i j}(t), v_{i j}(t), w_{i j}(t)\right]
$$

between telescopes $i$ and $j$ at time $t$

$$
V\left(u_{i j}(t), v_{i j}(t)\right) \equiv r_{i j}(t) .
$$

Note that we can obtain only a discrete set of $(u, v)$ sample points. Indeed, the number of instantaneous independent baselines between $p$ antennas is at most $\frac{1}{2} p(p-1)$. Also, using the Earth rotation, we have a finite set $\left\{t_{k}, k=1, \cdots, K\right\}$, where the number of epochs $K$ is given by the ratio of the observation time and the covariance averaging time (e.g., $K=12 \mathrm{~h} / 30 \mathrm{~s}=$ 1440 samples). The available sample coordinates $\left\{u_{i j, k}, v_{i j, k}\right\}$ give an irregular cover of the $(u, v)$ plane. For an East-West line array such as WSRT, the points lie on ellipses. A practical issue is the implementation of the geometrical delay compensation. It is usually introduced only at the back end of the receiver. At this point, also a phase correction is needed to compensate for the factor $e^{-2 \pi j w_{i j}(t)}$ in the measurement equation (2). This is referred to as fringe correction [39]. Since the Earth rotates, $w_{i j}(t)$ is slowly time varying, with a rate of change in the order of $0-10$ $\mathrm{Hz}$ depending on source declination and baseline length.

\section{B. Matrix Formulation}

For the discrete source model, we can now formulate our measurement equations in terms of matrices. Let $\boldsymbol{r}_{0}\left(t_{k}\right)$ be an arbitrary and time-varying reference point, typically at one of the elements of the array, and let us take the $(u, v, w)$ coordinates of the other telescopes with respect to this reference

$$
\boldsymbol{r}_{i}(t)-\boldsymbol{r}_{0}(t)=\lambda\left[u_{i 0}(t), v_{i 0}(t), w_{i 0}(t)\right], \quad i=1, \cdots, p .
$$

Equation (1) can then be written slightly differently as

$$
\begin{gathered}
V\left(\boldsymbol{r}_{i}(t), \boldsymbol{r}_{j}(t)\right)=\sum_{l=1}^{d} e^{-2 \pi_{\jmath} f \boldsymbol{s}_{l}^{T}\left(\boldsymbol{r}_{i}-\boldsymbol{r}_{0}\right) / c} I\left(\boldsymbol{s}_{l}\right) e^{2 \pi_{\jmath} f \boldsymbol{s}_{l}^{T}\left(\boldsymbol{r}_{j}-\boldsymbol{r}_{0}\right) / c} \\
\Leftrightarrow V\left(u_{i j}(t), v_{i j}(t)\right)=\sum_{l=1}^{d} e^{-2 \pi_{\jmath}\left(u_{i 0}(t) \ell_{l}+v_{i 0}(t) m_{l}\right)} I\left(\ell_{l}, m_{l}\right) \\
\cdot e^{2 \pi \jmath\left(u_{j 0}(t) \ell_{l}+v_{j 0}(t) m_{l}\right)} .
\end{gathered}
$$

In terms of correlation matrices, this equation can be written as $\boldsymbol{R}_{k}=A_{k} B A_{k}^{\mathrm{H}}$, where $\boldsymbol{R}_{k} \equiv \boldsymbol{R}\left(t_{k}\right)$

$$
\boldsymbol{A}_{k}=\left[\boldsymbol{a}_{k}\left(\ell_{1}, m_{1}\right), \cdots, \boldsymbol{a}_{k}\left(\ell_{d}, m_{d}\right)\right]
$$


and

$$
\begin{aligned}
\boldsymbol{a}_{k}(\ell, m) & =\left[\begin{array}{c}
e^{-2 \pi \jmath\left(u_{10}\left(t_{k}\right) \ell+v_{10}\left(t_{k}\right) m\right)} \\
\vdots \\
e^{-2 \pi \jmath\left(u_{p 0}\left(t_{k}\right) \ell+v_{p 0}\left(t_{k}\right) m\right)}
\end{array}\right] \\
\boldsymbol{B} & =\left[\begin{array}{ccc}
I\left(\ell_{1}, m_{1}\right) & 0 \\
& \ddots & \\
0 & & I\left(\ell_{d}, m_{d}\right)
\end{array}\right] .
\end{aligned}
$$

The vector function $\boldsymbol{a}_{k}(\ell, m)$ is called the array response vector in array signal processing. It describes the response of the telescope array to a source in the direction $(\ell, m)$. As usual, the array response is frequency-dependent. In this case, the response is also slowly time-varying due to the Earth rotation. Note, very importantly, that the function as shown here is completely known.

More realistically, the array response is less perfect. An important effect is that each antenna may have a different complex receiver gain $\gamma_{i}(t)$ dependent on many angle-independent effects such as cable losses, amplifier gains, and (slowly) varying atmospheric conditions. We also have to realize that most of the received signal consists of additive system noise. When this noise is zero-mean, independent among the antennas (thus spatially white), and identically distributed, then it has a covariance matrix that is a multiple of the identity matrix, $\sigma^{2} \boldsymbol{I}$, where $\sigma^{2}$ is the noise power on a single antenna inside the subband which we consider. Usually the noise is assumed to be Gaussian. The resulting model of the received covariance matrix then becomes

$$
\boldsymbol{R}_{k}=\boldsymbol{\Gamma}_{k} \boldsymbol{A}_{k} \boldsymbol{B} \boldsymbol{A}_{k}^{\mathrm{H}} \boldsymbol{\Gamma}_{k}^{\mathrm{H}}+\sigma^{2} \boldsymbol{I}
$$

where

$$
\boldsymbol{\Gamma}_{k}=\left[\begin{array}{ccc}
\gamma_{1, k} & & \mathbf{0} \\
& \ddots & \\
\mathbf{0} & & \gamma_{p, k}
\end{array}\right] .
$$

Note that this assumes that the noise is introduced after the varying receiver gains. This assumption is reasonable if the channels from the low-noise amplifier (LNA) outputs to the analog-to-digital converter (ADC) units are equal. Otherwise, it is still reasonable to assume that the noise is spatially white, i.e., the noise covariance matrix is diagonal. We can assume that the receivers noise power can be estimated using various calibration techniques; a simple diagonal scaling will then bring us back to the model (7).

\section{RF Interference}

Radio frequency interference (RFI) usually enters the antennas through the sidelobes of the main beam. It can be stronger or weaker than the system noise. An important property is that it has a certain directivity, so that it does not average out in the correlation process. Examples of harmful RFI are television broadcasts, geolocation satellites (GPS, GLONASS), taxi dispatch systems, airplane communication and navigation signals, wireless mobile communication (GSM), and satellite communication signals (Iridium). Thus interferers may be continuous or intermittent, narrowband or wideband, and strong or weak.

Suppose that we have a single interferer impinging onto the telescope array. The interfering signal reaches the array with different delays $\tau_{i}$ for each telescope. Assuming processing in narrow subbands as before, delays translate into phase shifts, ${ }^{3}$ and the received signal can be modeled as $x_{i}(t)=$ $a_{i} s(t) e^{-2 \pi J f \tau_{i}}$, or, in vector notation,

$$
\boldsymbol{x}(t)=\left[\begin{array}{c}
a_{1} e^{-2 \pi \jmath f_{c} \tau_{1}} \\
\vdots \\
a_{p} e^{-2 \pi \jmath f_{c} \tau_{p}}
\end{array}\right] s(t):=\boldsymbol{a} s(t) .
$$

Here, $s(t)$ is the baseband signal, and $a_{i}$ represents the telescope gain in the direction of the interferer, including any possible attenuation of the channel. Unlike much of the array signal processing literature, the $a_{i}$ are likely to be different for each telescope since the interferer is typically in the near field. This implies that it impinges on each telescope at a different angle, whereas the response of the telescopes is not omnidirectional. Hence, the corresponding array response vector $\boldsymbol{a}$ is now an unknown function. This vector is also called the spatial signature of the interfering source. It is slowly time varying, and we write $\boldsymbol{a}=\boldsymbol{a}(t)$.

Similarly, with $q$ interferers

$$
\begin{aligned}
\boldsymbol{x}(t)=\sum_{j=1}^{q} \boldsymbol{a}_{j}(t) s_{j}(t)=\boldsymbol{A}_{s}(t)\left[\begin{array}{c}
s_{1}(t) \\
\vdots \\
s_{q}(t)
\end{array}\right] \\
\boldsymbol{A}_{s}(t)=\left[\boldsymbol{a}_{1}(t), \cdots, \boldsymbol{a}_{q}(t)\right] .
\end{aligned}
$$

The subscript " $s$ " is used to distinguish $\boldsymbol{A}_{s}(t)$ from the array response matrix of the astronomical sources.

The corresponding correlation matrix at time $t_{k}$ is

$$
\boldsymbol{R}_{k}=\mathrm{E}\left\{\boldsymbol{x}\left(t_{k}\right) \boldsymbol{x}\left(t_{k}\right)^{\mathrm{H}}\right\}=\left(\boldsymbol{A}_{s}\right)_{k}\left(\boldsymbol{R}_{s}\right)_{k}\left(\boldsymbol{A}_{s}^{\mathrm{H}}\right)_{k} .
$$

The $q \times q$ matrix $\left(\boldsymbol{R}_{s}\right)_{k}=\mathrm{E}\left\{\boldsymbol{s}\left(t_{k}\right) \boldsymbol{s}^{\mathrm{H}}\left(t_{k}\right)\right\}$ depends on the correlational properties of the interfering signals. For independent interferers, it will be a diagonal matrix, with the $q$ interfering powers on the diagonal.

How well an empirical estimate $\hat{\boldsymbol{R}}_{\boldsymbol{k}}$ fits to $\boldsymbol{R}_{\boldsymbol{k}}$ depends on the stationarity of the scenario, and is open to discussion. For various reasons (mobile interferers with multipath fading, fixed interferers such as TV stations moving through the varying sidelobes of the rotating telescopes, fringe corrections of up to 10 $\mathrm{Hz}$ ), the stationarity of $a_{i}$ is often limited to about $10-100 \mathrm{~ms}$. In the rest of the paper, we make the assumption that indeed the available $\hat{\boldsymbol{R}}_{k}$ are obtained over stationary periods. In summary, the overall model including astronomical signals, array imperfections, interference, and noise is given by

$$
\begin{array}{r}
\boldsymbol{R}_{k}=\boldsymbol{\Gamma}_{k} \boldsymbol{A}_{k} B \boldsymbol{A}_{k}^{\mathrm{H}} \boldsymbol{\Gamma}_{k}+\left(\boldsymbol{A}_{s}\right)_{k}\left(\boldsymbol{R}_{s}\right)_{k}\left(\boldsymbol{A}_{s}\right)_{k}^{\mathrm{H}}+\sigma^{2} \boldsymbol{I}, \\
k=0,1, \cdots
\end{array}
$$

${ }^{3}$ For this, the processing bandwidths should be much less than the inverse of the maximal delay. For example, in WSRT the largest baseline is $3000 \mathrm{~m}$, corresponding to a maximal delay of $10 \mu \mathrm{s}$. Hence the narrowband assumption holds for bandwidths less than $100 \mathrm{kHz}$ [21]. 
where we assume that the interference term $\boldsymbol{A}_{s}$ is unstructured, and $\operatorname{rank}\left(A_{s}\right)=q<p$.

\section{SPATIAL FILTERING}

An on-line interference mitigation system will consist of two stages. As a first step the presence of interference is detected. This part is considered in [21] and [18] and is demonstrated on astronomical data in [20]. In the case of continuous interference it is reasonable to use its spatial signature in order to remove it. This leads to spatial filtering techniques.

\section{A. Projecting out the Interferer}

Let us assume that we have obtained a covariance matrix $\boldsymbol{R}$, which contains the rather weak covariance matrix of the astronomical sources (visibilities) $\boldsymbol{R}_{v}=\boldsymbol{\Gamma}_{k} \boldsymbol{A}_{k} \boldsymbol{B} \boldsymbol{A}_{k}^{\mathrm{H}} \boldsymbol{\Gamma}_{k}$ plus white noise. ${ }^{4}$ Suppose also that there is an interferer with power $\sigma_{s}^{2}$

$$
\boldsymbol{R}=\boldsymbol{R}_{v}+\sigma_{s}^{2} \boldsymbol{a} \boldsymbol{a}^{\mathrm{H}}+\sigma^{2} \boldsymbol{I}
$$

Assuming that $\boldsymbol{a}$ is known it is possible to null all energy with spatial signature $\boldsymbol{a}$. To this end, we can introduce the projection matrices

$$
P_{\boldsymbol{a}}=\boldsymbol{a}\left(\boldsymbol{a}^{\mathrm{H}} \boldsymbol{a}\right)^{-1} \boldsymbol{a}^{\mathrm{H}} \quad P_{\boldsymbol{a}}^{\perp}=I-\boldsymbol{a}\left(\boldsymbol{a}^{\mathrm{H}} \boldsymbol{a}\right)^{-1} \boldsymbol{a}^{\mathrm{H}} .
$$

It is easily seen that $\boldsymbol{P}_{\boldsymbol{a}}^{\perp} \boldsymbol{a}=0$, so that if we apply $\boldsymbol{P}_{\boldsymbol{a}}^{\perp}$ as a spatial filter to $R$, we obtain

$$
\boldsymbol{R} t:=\boldsymbol{P}_{\boldsymbol{a}}^{\perp} \boldsymbol{R} \boldsymbol{P}_{\boldsymbol{a}}^{\perp}=\boldsymbol{P}_{\boldsymbol{a}}^{\perp} \boldsymbol{R}_{v} \boldsymbol{P}_{\boldsymbol{a}}^{\perp}+\sigma^{2} \boldsymbol{P}_{\boldsymbol{a}}^{\perp} .
$$

Thus the interference is completely removed. At the same time, the visibility matrix is modified by the projections, and the noise is not white anymore since one dimension is missing. The imaging stage has to be aware of this, which is the topic of Section V.

This idea is also applicable to multiple narrowband interferers, and we do not need to know the spatial signatures of the interferers in advance. Indeed, if the total number of interferers inside a subband is $q<p$, an eigenvalue decomposition allows to estimate the corresponding "interference subspace" spanned by the spatial signatures from the data covariance matrix, and subsequently project out this subspace.

Thus let $\boldsymbol{R}=\boldsymbol{U} \boldsymbol{\Lambda} \boldsymbol{U}^{\mathrm{H}}$ be the eigendecomposition of $\boldsymbol{R}$. For the purpose of interference cancellation we assume that the sky sources are weak: $\boldsymbol{R}_{v} \ll \sigma^{2} I$, and thus their influence can be ignored in the eigendecomposition. Let $\boldsymbol{U}=\left[\boldsymbol{U}_{s} \boldsymbol{U}_{n}\right]$ where $\boldsymbol{U}_{s}$ is $p \times q$ and contains the eigenvectors corresponding to the $q$ largest eigenvalues, and $U_{n}$ collects the remaining eigenvectors. In the noise-free case, $R$ has rank $q$ and

$$
\boldsymbol{R}=\boldsymbol{A}_{s} \boldsymbol{R}_{s} \boldsymbol{A}_{s}^{\mathrm{H}}=\boldsymbol{U}_{s} \boldsymbol{\Lambda} \boldsymbol{U}_{s}^{\mathrm{H}} .
$$

In the noisy case

$$
\boldsymbol{R}=\boldsymbol{A}_{s} \boldsymbol{R}_{s} \boldsymbol{A}_{s}^{\mathrm{H}}+\sigma^{2} \boldsymbol{I}
$$

${ }^{4}$ In this section we consider a single covariance matrix hence we drop the index $k$. with eigenvalue decomposition

$$
\begin{aligned}
\boldsymbol{R} & =\boldsymbol{U}_{s} \boldsymbol{\Lambda}_{s} \boldsymbol{U}_{s}^{\mathrm{H}}+\sigma^{2}\left[\begin{array}{ll}
\boldsymbol{U}_{s} & \boldsymbol{U}_{n}
\end{array}\right]\left[\begin{array}{ll}
\boldsymbol{U}_{s} & \boldsymbol{U}_{n}
\end{array}\right]^{\mathrm{H}} \\
& =\left[\begin{array}{ll}
\boldsymbol{U}_{s} & \boldsymbol{U}_{n}
\end{array}\right]\left[\begin{array}{c|c}
\boldsymbol{\Lambda}_{s}+\sigma^{2} \boldsymbol{I}_{q} & 0 \\
\hline 0 & \sigma^{2} \boldsymbol{I}_{p-q}
\end{array}\right]\left[\begin{array}{c}
\boldsymbol{U}_{s}^{\mathrm{H}} \\
\boldsymbol{U}_{n}^{\mathrm{H}}
\end{array}\right] .
\end{aligned}
$$

Therefore, the smallest eigenvalue $\left(\sigma^{2}\right)$ has multiplicity $p-q$, and

$$
\operatorname{span}\left(\boldsymbol{U}_{s}\right)=\operatorname{span}\left(\boldsymbol{A}_{s}\right) \quad \boldsymbol{U}_{n}^{\mathrm{H}} A_{s}=0 .
$$

We refer to $\boldsymbol{U}_{s}$ as the interference subspace. According to (12), $\boldsymbol{A}_{s} \perp \boldsymbol{U}_{n}$, so that $\boldsymbol{P}_{\boldsymbol{A}_{s}}^{\perp}=\boldsymbol{U}_{n} \boldsymbol{U}_{n}^{\mathrm{H}}$. Thus the eigenvalue decomposition of $\boldsymbol{R}$ allows us to detect the number of interferers (from the number of repeated small eigenvalues) and to identify the projection matrix $\boldsymbol{P}_{\boldsymbol{A}_{s}}^{\perp}=\boldsymbol{U}_{n} \boldsymbol{U}_{n}^{\mathrm{H}}$ to project them out, as in (10). Note that we do not have to know $A_{s}$. This hinges upon the fact that the noise covariance is white (in general, known), and the visibility matrix $\boldsymbol{R}_{v}$ is insignificant at these time scales (otherwise, it might disturb the eigenvalue decomposition).

In practice, we only have a sample estimate $\hat{R}$ of $\boldsymbol{R}$. The eigenvalue decomposition of this matrix

$$
\hat{\boldsymbol{R}}=\hat{\boldsymbol{U}}_{s} \hat{\boldsymbol{\Lambda}}_{s} \hat{\boldsymbol{U}}_{s}^{\mathrm{H}}+\hat{\boldsymbol{U}}_{n} \hat{\boldsymbol{\Lambda}}_{n} \hat{\boldsymbol{U}}_{n}^{\mathrm{H}}
$$

gives an ML estimate of $\boldsymbol{U}_{n}$ [2].

One might be worried that if we use the estimated subspace for the projections, it might leave correlated residual interference components that eventually will show up in the final image. This is in fact not the case, as we will now demonstrate that the residual interference is spatially white.

\section{B. Residual Interference After Projections}

A perturbation analysis of the eigenvalue decomposition yields asymptotic expressions for the residual interference in the covariance matrices after spatial filtering. To this end, we utilize the following theorem from [40], a proof of which is given in [36].

Theorem IV.1: Let $\hat{\boldsymbol{R}}$ be the sample covariance matrix based on $N$ samples of a $p$-dimensional complex Gaussian random process, with zero mean and covariance $\boldsymbol{R}$. Let $\boldsymbol{R}=\boldsymbol{U} \Lambda U^{\mathrm{H}}$ be an eigenvalue decomposition of $\boldsymbol{R}$, with $\boldsymbol{U}=\left[\boldsymbol{u}_{1}, \cdots, \boldsymbol{u}_{p}\right]$ unitary, and $\boldsymbol{\Lambda}=\operatorname{diag}\left[\lambda_{1}, \cdots, \lambda_{p}\right]$, where $\lambda_{1}>\cdots>\lambda_{q}>$ $\sigma^{2}$ and $\lambda_{q+1}=\cdots=\lambda_{p}=\sigma^{2}$. Let $\boldsymbol{U}_{s}=\left[\boldsymbol{u}_{1}, \cdots, \boldsymbol{u}_{q}\right]$. Then for $m>q$, we have that $\boldsymbol{P}_{\boldsymbol{U}_{s}} \hat{\boldsymbol{u}}_{m}$ is asymptotically a zero-mean Gaussian random process with variance determined by

$$
\begin{aligned}
\mathrm{E} & \left\{\left(\boldsymbol{P}_{\boldsymbol{U}_{s}} \hat{\boldsymbol{u}}_{i}\right)\left(\boldsymbol{P}_{\boldsymbol{U}_{s}} \hat{\boldsymbol{u}}_{j}\right)^{\mathrm{H}}\right\} \\
& =\frac{\sigma^{2}}{N}\left[\sum_{n=1}^{q} \frac{\lambda_{n}}{\left(\lambda_{n}-\sigma^{2}\right)^{2}} \boldsymbol{u}_{n} \boldsymbol{u}_{n}^{\mathrm{H}}\right] \delta_{i, j}+o\left(N^{-1}\right), \quad i, j>q .
\end{aligned}
$$

For simplicity, let us specialize to the case of $q=1$ narrowband interferer, with power $\sigma_{s}^{2}$ per sample and spatial sig- 
nature $\boldsymbol{a}$ normalized to $\|\boldsymbol{a}\|^{2}=p$. In this case, $\lambda_{1}=p \sigma_{s}^{2}+\sigma^{2}$, $\boldsymbol{u}_{1}=\boldsymbol{a} /\|\boldsymbol{a}\|=\boldsymbol{a} / \sqrt{p}$, and (13) gives

$$
\mathrm{E}\left\{\left\|\boldsymbol{P}_{\boldsymbol{u}_{1}} \hat{\boldsymbol{u}}_{m}\right\|^{2}\right\}=\frac{\sigma^{2}}{N} \frac{p \sigma_{s}^{2}+\sigma^{2}}{\left(p \sigma_{s}^{2}\right)^{2}}, \quad m>1 .
$$

Now note that

$$
\begin{aligned}
\left\|\boldsymbol{P}_{\boldsymbol{u}_{1}} \hat{\boldsymbol{u}}_{m}\right\|^{2} & =\left\|\boldsymbol{u}_{1} \boldsymbol{u}_{1}^{\mathrm{H}} \hat{\boldsymbol{u}}_{m}\right\|^{2}=\left\|\boldsymbol{u}_{1}^{\mathrm{H}} \hat{\boldsymbol{u}}_{m}\right\|^{2} \\
& =\left\|\hat{\boldsymbol{u}}_{m} \hat{\boldsymbol{u}}_{m}^{\mathrm{H}} \boldsymbol{u}_{1}\right\|^{2}=\left\|\boldsymbol{P}_{\hat{\boldsymbol{u}}_{m}} \boldsymbol{u}_{1}\right\|^{2}
\end{aligned}
$$

so that

$$
\mathrm{E}\left\{\left\|\boldsymbol{P}_{\hat{\boldsymbol{u}}_{m}} \boldsymbol{a}\right\|^{2}\right\}=p \mathrm{E}\left\{\left\|\boldsymbol{P}_{\hat{\boldsymbol{u}}_{m}} \boldsymbol{u}_{1}\right\|^{2}\right\}=p \mathrm{E}\left\{\left\|\boldsymbol{P}_{\boldsymbol{u}_{1}} \hat{u}_{m}\right\|^{2}\right\} .
$$

Similarly, we get for $m \neq n$

$$
\mathrm{E}\left\{\left(\boldsymbol{P}_{\hat{\boldsymbol{u}}_{m}} \boldsymbol{u}_{1}\right)^{\mathrm{H}}\left(\boldsymbol{P}_{\hat{\boldsymbol{u}}_{n}} \boldsymbol{u}_{1}\right)\right\}=0 .
$$

If we define the input Interference-to-Noise Ratio (INR) as $\mathrm{INR}=\sigma_{s}^{2} / \sigma^{2}$, then we finally obtain

$$
\begin{aligned}
& \mathrm{E}\left\{\left\|\boldsymbol{P}_{\hat{\mathbf{u}}_{m}} \boldsymbol{a}\right\|^{2}\right\}=p \frac{\sigma^{2}}{N} \frac{p \sigma_{s}^{2}+\sigma^{2}}{\left(p \sigma_{s}^{2}\right)^{2}}=\frac{p}{N} \frac{p \mathrm{INR}+1}{(p \mathrm{INR})^{2}}, \quad m>1 \\
& \Rightarrow \mathrm{E}\left\{\left\|\boldsymbol{P}_{\hat{\boldsymbol{a}}}^{\perp} \boldsymbol{a}\right\|^{2}\right\}=\mathrm{E}\left\{\left\|\boldsymbol{P}_{\hat{\boldsymbol{u}}_{1}}^{\perp} \boldsymbol{a}\right\|^{2}\right\}=\frac{p(p-1)}{N} \frac{p \mathrm{INR}+1}{(p \mathrm{INR})^{2}} .
\end{aligned}
$$

Let us assume that the estimate $\hat{\boldsymbol{a}}_{1}$ is approximately independent of the interfering signal. The residual INR at the output after spatial filtering is then

$$
\mathrm{INR}^{\prime}:=\frac{\sigma_{s}^{2}}{\sigma^{2}} \frac{\mathrm{E}\left\{\left\|\boldsymbol{P}_{\hat{\boldsymbol{a}}_{1}}^{\perp} \boldsymbol{a}\right\|^{2}\right\}}{p-1}=\frac{1}{N}\left(1+\frac{1}{p \mathrm{INR}}\right) .
$$

These expressions are very satisfactory. Indeed, note from (14) that the expected residual interference power is the same in each of the directions $\hat{\boldsymbol{u}}_{m}$, which together form an orthonormal basis of the projected space. This means that the residual interference is spatially white within the projected space (up to second order (in $\frac{1}{N}$ ) effects), and only increases the effective noise power without adding spatial features to it. The effective noise power at the output is

$$
\left(\sigma^{\prime}\right)^{2}=\sigma^{2}\left\{1+\frac{1}{N}\left(1+\frac{1}{p \mathrm{INR}}\right)\right\} .
$$

Fig. 2 shows the residual interference in a simulation, for $N=100$ samples and $p=8$ antennas. The reference lines are given by the predicted value in (16), and the line INR' $\equiv$ INR. Although the predicted value fits very well for sufficiently large INR, it is seen that for small INR, (16) loses its validity. This is because Theorem IV.1 is valid only for eigenvalues sufficiently above the noise power. For small INR's, the estimated interference subspace will be a random vector, and the projection will have no effect on the INR. The crossover point is approximately given by INR $=\frac{1}{N}+\frac{1}{\sqrt{N p}}$. The generalization for higher dimensional interference subspace is straightforward using the orthogonality of the interference eigenvectors. Note, however, that

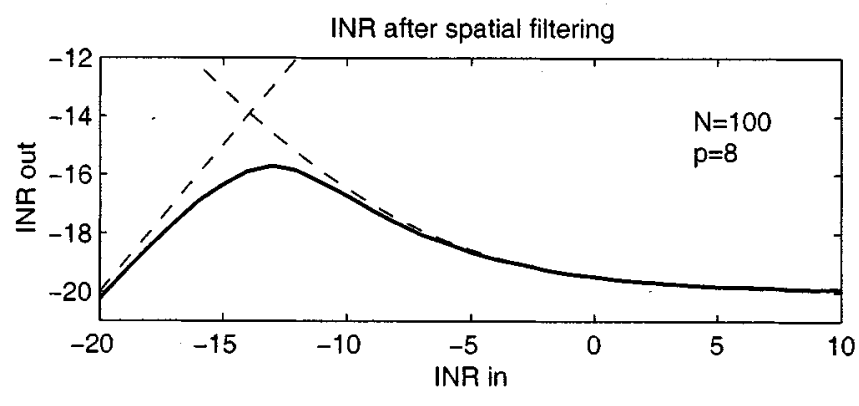

Fig. 2. Residual INR after spatial filtering.

in this case the eigenvectors lose their natural interpretation as spatial signatures of the various interferers.

\section{Other Spatial Filtering Possibilities}

Without going into too much detail, we mention a few other possibilities for spatial filtering and interference cancellation. Suppose there is a single interferer

$$
\boldsymbol{R}=\boldsymbol{R}_{v}+\sigma_{s}^{2} \boldsymbol{a} \boldsymbol{a}^{\mathrm{H}}+\sigma^{2} \boldsymbol{I} .
$$

Subtraction: With an estimate of $\boldsymbol{a}$ and an estimate of its power, we can try to subtract it from the covariance data

$$
\tilde{\boldsymbol{R}}=\boldsymbol{R}-\hat{\sigma}_{s}^{2} \hat{\boldsymbol{a}} \hat{\boldsymbol{a}}^{\mathrm{H}} .
$$

Without other knowledge, the best estimate of $\boldsymbol{a}$ is the dominant eigenvector $\boldsymbol{u}_{1}$ of $\boldsymbol{R}$, and likewise the best estimate of $\sigma_{s}^{2}$ is $\lambda_{1}-\sigma^{2}$. Since both of these are derived from $R$, it turns out to be not too different from the projection scheme. Indeed, if we look at

$$
\left(\boldsymbol{I}-\alpha \boldsymbol{u}_{1} \boldsymbol{u}_{1}^{\mathrm{H}}\right) \boldsymbol{R}\left(\boldsymbol{I}-\alpha \boldsymbol{u}_{1} \boldsymbol{u}_{1}^{\mathrm{H}}\right)=\boldsymbol{R}-\boldsymbol{u}_{1} \boldsymbol{u}_{1}^{\mathrm{H}} \lambda_{1}\left(2 \alpha-\alpha^{2}\right)
$$

we can make it equal to (17) by selecting $\alpha$ such that $\lambda_{1}\left(2 \alpha-\alpha^{2}\right)=\hat{\sigma}_{s}^{2}$. The projection scheme had $\alpha=1$.

Spatial Whitening: In this scheme, we try to make the interference plus noise white again. This component is equal to $\sigma_{s}^{2} \boldsymbol{a} \boldsymbol{a}^{\mathrm{H}}+\sigma^{2} \boldsymbol{I}$, so we pre- and postmultiply with square-root factors of it

$$
\begin{aligned}
\tilde{\boldsymbol{R}} & =\left(\hat{\sigma}_{s}^{2} \hat{\boldsymbol{a}} \hat{\boldsymbol{a}}^{\mathrm{H}}+\hat{\sigma}^{2} \boldsymbol{I}\right)^{-1 / 2} \boldsymbol{R}\left(\hat{\sigma}_{s}^{2} \hat{\boldsymbol{a}} \hat{\boldsymbol{a}}^{\mathrm{H}}+\hat{\sigma}^{2} \boldsymbol{I}\right)^{-1 / 2} \\
& =(\cdot)^{-1 / 2} \boldsymbol{R}_{v}(\cdot)^{-1 / 2}+\boldsymbol{I} .
\end{aligned}
$$

Subtraction of a Reference Signal: If we have a reference antenna that receives a "clean" copy of the interfering signal, then we might try to subtract this reference signal from the telescope signals. There are many adaptive schemes for doing so, e.g., LMS or RLS. This solution involves a separate receiver for each interferer. To shorten the filter lengths subband processing is recommended, as otherwise the adaptation might be slow for wideband signals. This filtering scheme is similar to the first-mentioned subtraction scheme, except that the spatial signature $\hat{\boldsymbol{a}}$ of the interferer is computed from correlations with the reference antenna. 
Note that each of these filtering schemes can be described as a linear operation on the entries of the observed data covariance matrix $\hat{\boldsymbol{R}}_{k}$. In future equations, we will denote the linear operation by $\boldsymbol{L}_{k}$, and the output after filtering $\tilde{\boldsymbol{R}}_{k}=L_{k} \boldsymbol{R}_{k} \boldsymbol{L}_{k}^{\mathrm{H}}$.

\section{Fourier IMAGing AfTER SPATIAL FILTERING}

In the previous sections, we discussed spatial filtering techniques. It was shown that an attractive scheme for removing the interference is by projecting it out. However, by doing so we replace the observed visibilities $V\left(u_{i}, v_{i}\right)$ in the matrix $\boldsymbol{R}_{v}$, by some (known) linear combination. In this section, we discuss the implications of this for the imaging.

\section{A. Classical Inverse Fourier Imaging}

The relation between sky brightness $I(\ell, m)$ and visibilities $V(u, v)$ (where $u, v$ are taken at frequency $f$ ) is

$$
V(u, v)=\iint I(\ell, m) e^{-2 \pi \jmath(u \ell+v m)} d \ell d m .
$$

We have measured $V$ on a discrete set of baselines $\left\{\left(u_{i}, v_{i}\right)\right\}$. The "dirty image" (a lumpy image obtained via direct Fourier inversion possibly modified with some weights $c_{i}$ ) is defined by

$$
I_{D}(\ell, m):=\sum_{i} c_{i} V\left(u_{i}, v_{i}\right) e^{2 \pi j\left(u_{i} \ell+v_{i} m\right)} .
$$

It is equal to the two-dimensional (2-D) convolution of the true image $I$ with a point spread function known as the "dirty beam"

$$
\begin{aligned}
I_{D}(\ell, m)= & \sum_{i} c_{i} V\left(u_{i}, v_{i}\right) e^{2 \pi \jmath\left(u_{i} \ell+v_{i} m\right)} \\
= & \sum_{i} c_{i}\left[\iint I\left(\ell^{\prime}, m^{\prime}\right) e^{-2 \pi \jmath\left(u_{i} \ell^{\prime}+v_{i} m^{\prime}\right)} d \ell^{\prime} d m^{\prime}\right] \\
& \cdot e^{2 \pi \jmath\left(u_{i} \ell+v_{i} m\right)} \\
= & \iint I\left(\ell^{\prime}, m^{\prime}\right)\left[\sum_{i} c_{i} e^{2 \pi \jmath\left(u_{i}\left(\ell-\ell^{\prime}\right)+v_{i}\left(m-m^{\prime}\right)\right)}\right] \\
& \cdot d \ell^{\prime} d m^{\prime} \\
= & \iint I\left(\ell^{\prime}, m^{\prime}\right) B_{0}\left(\ell-\ell^{\prime}, m-m^{\prime}\right) d \ell^{\prime} d m^{\prime}
\end{aligned}
$$

or

$$
I_{D}=I * B_{0}, \quad B_{0}(\ell, m):=\sum_{i} c_{i} e^{2 \pi_{\jmath}\left(u_{i} \ell+v_{i} m\right)} .
$$

$B_{0}$ is the dirty beam, centered at the origin. The weights $\left\{c_{i}\right\}$ are arbitrary coefficients designed to obtain an acceptable beam shape, with low sidelobes, in spite of the irregular sampling.

Specializing to a point source model

$$
I(\ell, m)=\sum_{l} I_{l} \delta\left(\ell-\ell_{l}, m-m_{l}\right)
$$

where $I_{l}$ is the intensity of the source at location $\left(\ell_{l}, m_{l}\right)$, gives

$$
\begin{aligned}
V(u, v) & =\sum_{l} I_{l} e^{-2 \pi \jmath\left(u \ell_{l}+v m_{l}\right)} \\
I_{D}(\ell, m) & =\sum_{l} I_{l} B_{0}\left(\ell-\ell_{l}, m-m_{l}\right) .
\end{aligned}
$$

TABLE I

THE CLEAN ALGORITHM

$l=0$

while $I_{D}$ is not noise-like:

$$
\left[\begin{array}{l}
\left(\ell_{l}, m_{l}\right)=\arg \max I_{D}(\ell, m) \\
\lambda_{l}=I_{D}\left(\ell_{l}, m_{l}\right) / B_{0}(0,0) \\
I_{D}:=I_{D}-\gamma \lambda_{l} B_{0}\left(\ell-\ell_{l}, m-m_{l}\right) \\
l=l+1
\end{array}\right.
$$

$I=I_{D}+\sum_{l} \gamma \lambda_{l} B_{\text {synth }}\left(\ell-\ell_{l}, m-m_{l}\right)$

Thus every point source excites the dirty beam centered at its location $\left(\ell_{l}, m_{l}\right)$.

From the dirty image $I_{D}$ and the known dirty beam $B_{0}$, the desired image $I$ is obtained via a deconvolution process. A popular method for doing this is the CLEAN algorithm [12]. The algorithm assumes that $B_{0}$ has its peak at the origin, and consists of a loop in which a candidate location $\left(\ell_{l}, m_{l}\right)$ is selected as the largest peak in $I_{D}$, and subsequently a small multiple of $B_{0}\left(\ell-\ell_{l}, m-m_{l}\right)$ is subtracted from $I_{D}$. The objective is to minimize the residual, until it converges to the noise level. A short description of the algorithm is given in Table I. The parameter $\gamma \leq 1$ is called the loop gain and serves the purpose of interpolation over the grid, $\lambda_{l}$ is the estimated power of the source.

\section{B. Inverse Fourier Imaging After Projections}

If we take projections or any other linear combination $\left[c_{i j}\right]$ of the visibilities $\left\{V\left(u_{i}, v_{i}\right)\right\}$ during measurements, as in Section IV, we have instead available

$$
Z\left(u_{i}, v_{i}\right)=\sum_{j} c_{i j} V\left(u_{j}, v_{j}\right)
$$

The coefficients $c_{i j}$ are connected to the linear operations $\left\{\boldsymbol{L}_{k}\right\}$ of Section IV, and $Z\left(u_{i}, v_{i}\right)$ are the samples contained in the collection $\left\{\tilde{\boldsymbol{R}}_{k}\right\}$.

Suppose we compute the dirty image in the same way as before, but now from $Z$

$$
\begin{aligned}
I_{D}(\ell, m) & :=\sum_{i} Z\left(u_{i}, v_{i}\right) e^{2 \pi \jmath\left(u_{i} \ell+v_{i} m\right)} \\
& =\sum_{i} \sum_{j} c_{i j} V\left(u_{j}, v_{j}\right) e^{2 \pi \jmath\left(u_{i} \ell+v_{i} m\right)} .
\end{aligned}
$$

Then

$$
\begin{array}{r}
I_{D}(\ell, m)=\sum_{i} \sum_{j} c_{i j} V\left(u_{j}, v_{j}\right) e^{2 \pi \jmath\left(u_{i} \ell+v_{i} m\right)} \\
=\sum_{i} \sum_{j} c_{i j}\left[\iint I\left(\ell^{\prime}, m^{\prime}\right) e^{-2 \pi \jmath\left(u_{j} \ell^{\prime}+v_{j} m^{\prime}\right)}\right. \\
\left.\cdot d \ell^{\prime} d m^{\prime}\right] e^{j\left(u_{i} \ell+v_{i} m\right)}
\end{array}
$$




$$
\begin{gathered}
=\iint I\left(\ell^{\prime}, m^{\prime}\right)\left[\sum_{i} \sum_{j} c_{i j} e^{-2 \pi \jmath\left(u_{j} \ell^{\prime}+v_{j} m^{\prime}\right)}\right. \\
\left.\cdot e^{2 \pi \jmath\left(u_{i} \ell+v_{i} m\right)}\right] d \ell^{\prime} d m^{\prime} \\
=\iint I\left(\ell^{\prime}, m^{\prime}\right) B\left(\ell, m, \ell^{\prime}, m^{\prime}\right) d \ell^{\prime} d m^{\prime}
\end{gathered}
$$

where

$$
B\left(\ell, m, \ell^{\prime}, m^{\prime}\right):=\sum_{i} \sum_{j} c_{i j} e^{-2 \pi \jmath\left(u_{j} \ell^{\prime}+v_{j} m^{\prime}\right)} e^{2 \pi \jmath\left(u_{i} \ell+v_{i} m\right)} .
$$

Thus the dirty image is again obtained via a convolution, but the dirty beam is now space-varying. $B\left(\ell, m, \ell^{\prime}, m^{\prime}\right)$ is a beam centered at $\left(\ell^{\prime}, m^{\prime}\right)$ and measured at $(\ell, m)$.

With a point-source model,

$$
I_{D}(\ell, m)=\sum_{l} I_{l} B\left(\ell, m, \ell_{l}, m_{l}\right)=\sum_{l} I_{l} B_{l}(\ell, m)
$$

where

$$
B_{l}(\ell, m):=\sum_{i} \sum_{j} c_{i j} e^{-2 \pi \jmath\left(u_{j} \ell_{l}+v_{j} m_{l}\right)} e^{2 \pi \jmath\left(u_{i} \ell+v_{i} m\right)} .
$$

Again, every point source excites a beam centered at its location $\left(\ell_{l}, m_{l}\right)$, but the beams may all be different: they are space-varying. Nonetheless, they are completely known if we know the linear combinations that we took during observations. Thus the CLEAN algorithm in Table I can readily be modified to take the varying beam shapes into account: simply replace $B_{0}(\ell, m)$ by $B_{l}(\ell, m)$ everywhere in the algorithm. Some remaining issues are as follows.

1) It is not a priori guaranteed that the main peak of $B_{l}(\ell, m)$ is indeed centered at $\left(\ell_{l}, m_{l}\right)$.

2) The noise is not necessarily white, and the coloring should be taken into account.

3) The computational complexity is increased since we have to construct $B_{l}(\ell, m)$ for every point $\left(\ell_{l}, m_{l}\right)$.

The first two points are addressed in Section VI-C.

To demonstrate the spatial variation effect of the dirty beam, we have generated an unfiltered dirty beam $B_{0}(\ell, m)$ (Fig. 3), and the beams $B\left(\ell, m, \ell^{\prime}, m^{\prime}\right)$ that would result after projecting out an interferer with a fixed terrestrial location. We show the latter for a source located at the center of the field, i.e., $B(\ell, m, 0,0)$ (Fig. 4$)$, and for a source at $\left(40^{\prime \prime}, 30^{\prime \prime}\right)$, i.e., $B\left(\ell, m, 40^{\prime \prime}, 30^{\prime \prime}\right)$ (Fig. 5). ${ }^{5}$ Note that the spatial projections have modified the shape of the beam, in particular the sidelobes, and that the response is not constant but varies with the location of the source. Although the changes do not look very dramatic, the differences are in fact important: accurate knowledge of the beam shapes is essential in the deconvolution step, especially if weak sources are to be detected among sources that are orders of magnitude stronger.

\section{IMAGING VIA BEAMFORMING TECHNIQUES}

In this section, we reformulate the classical inverse-Fourier imaging technique and the CLEAN algorithm for deconvolu-

\footnotetext{
${ }^{5}$ The beams have been computed for $\left(u_{i}, v_{i}\right)$ samples corresponding to a WSRT antenna configuration with $p=14$ telescopes and $K=100$ covariance epochs over $12 \mathrm{~h}$.
}

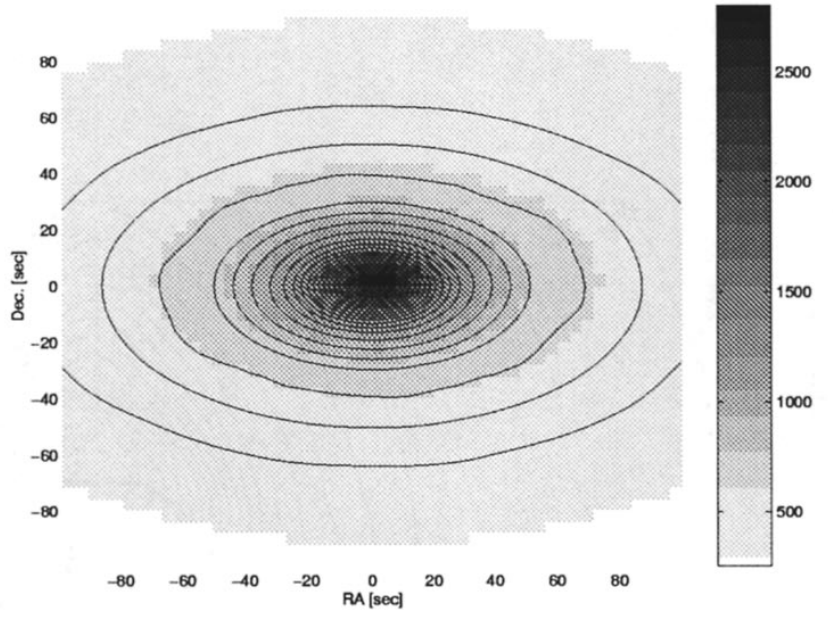

Fig. 3. Classical dirty beam $B_{0}(\ell, m)$, no interference suppression.

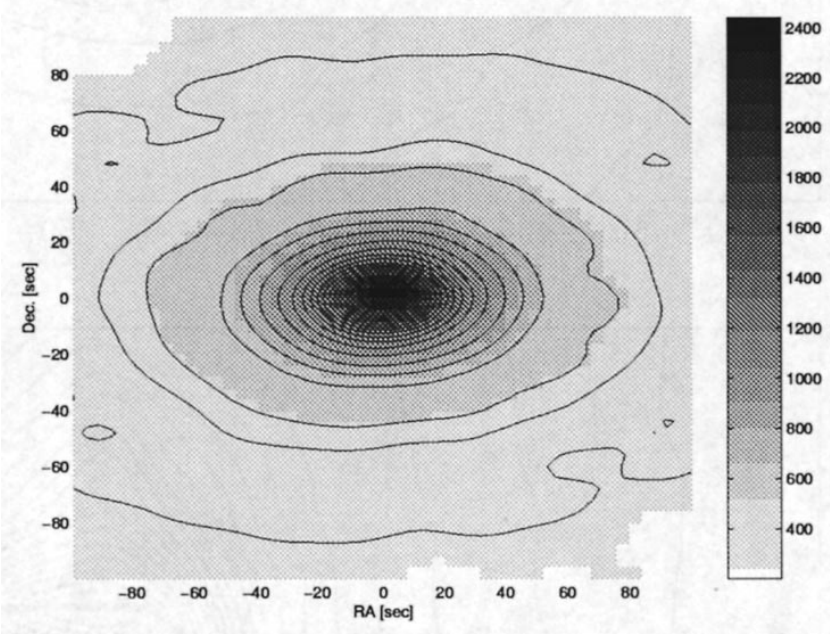

Fig. 4. Dirty beam resulting after spatial filtering. Beam $B(\ell, m, 0,0)$ for a source at the center of the field view.

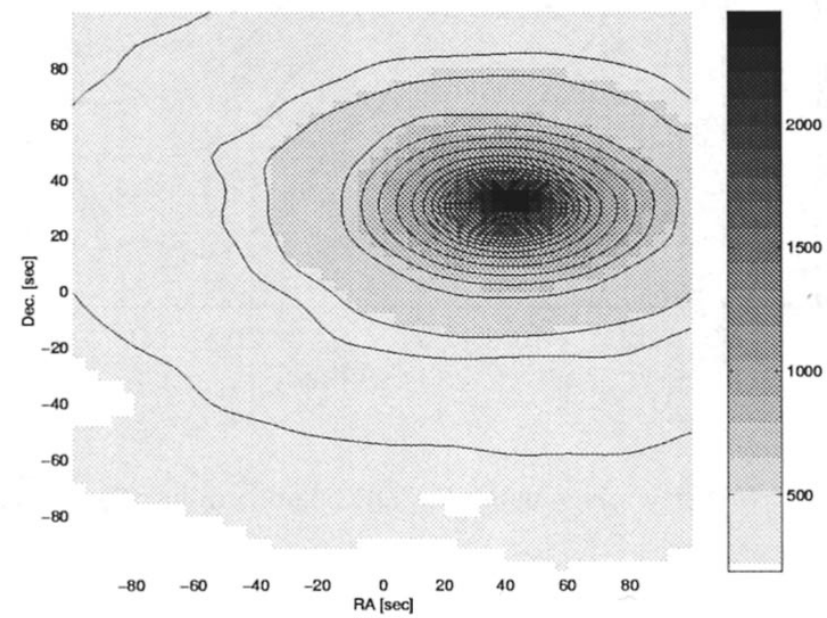

Fig. 5. Dirty beam resulting after spatial filtering. Beam $B\left(\ell, m, 40^{\prime \prime}, 30^{\prime \prime}\right)$ for a source at $\left(40^{\prime \prime}, 30^{\prime \prime}\right)$.

tion in terms of a more general iterative beamforming procedure. This is possible since we have a parametric point-source 
model, and the prime objective of the deconvolution step is to estimate the location of the point sources. The interpretation of the deconvolution problem as one of DOA estimation allows access to a potentially large number of algorithms that have been developed for this application.

\section{A. CLEAN and Sequential Beamforming}

We set out by showing how CLEAN can be interpreted as a sequential beamforming procedure.

Let us assume that we have available a collection of measured covariance matrices $\hat{\boldsymbol{R}}_{k}$, obtained at times $t_{k}$ with $k=$ $1, \cdots, K$, and let us assume the parametric model of (7), i.e.,

$$
\boldsymbol{R}_{k}=\boldsymbol{A}_{k} \boldsymbol{B} \boldsymbol{A}_{k}^{\mathrm{H}}+\sigma^{2} \boldsymbol{I} \text {. }
$$

Here, the unknown parameters are the source locations $\boldsymbol{s}_{l}=$ $\left(\ell_{l}, m_{l}\right), l=1, \cdots, d$ in each of the $A_{k}$, and the source brightness $I_{l}$ in $\boldsymbol{B}$. A natural formulation for the estimation of these parameters is to pose it as the solution of an LS cost function, given by

$$
\begin{aligned}
{\left[\left\{\hat{\boldsymbol{s}}_{l}\right\}, \hat{\boldsymbol{B}}\right]=\underset{\left\{\boldsymbol{s}_{l}\right\}, \boldsymbol{B}}{\operatorname{argmin}} } & \sum_{k=1}^{K} \\
& \left\|\hat{\boldsymbol{R}}_{k}-\boldsymbol{A}_{k}\left(\left\{\boldsymbol{s}_{l}\right\}\right) \boldsymbol{B} \boldsymbol{A}_{k}^{\mathrm{H}}\left(\left\{\boldsymbol{s}_{l}\right\}\right)-\sigma^{2} I\right\|_{F}
\end{aligned}
$$

( $\boldsymbol{B}$ is constrained to be diagonal with positive entries). This is recognized as the same model as used for DOA estimation in array processing. Note, however, that the array is moving ( $A_{k}$ is time-dependent), and that there are many more sources than the dimension of each covariance matrix.

In this notation, the image formation in Section $\mathrm{V}-\mathrm{A}$ can be formulated as follows. Recall from (5) and (6) that

$$
\begin{gathered}
V\left(u_{i j}\left(t_{k}\right), v_{i j}\left(t_{k}\right)\right) \equiv r_{i j}\left(t_{k}\right) \equiv\left(\boldsymbol{R}_{k}\right)_{i j}, \quad k=1, \cdots, K \\
\boldsymbol{a}_{k}(\ell, m)=\left[\begin{array}{c}
e^{-2 \pi \jmath\left(u_{10}\left(t_{k}\right) \ell+v_{10}\left(t_{k}\right) m\right)} \\
\vdots \\
e^{-2 \pi \jmath\left(u_{p 0}\left(t_{k}\right) \ell+v_{p 0}\left(t_{k}\right) m\right)}
\end{array}\right], \quad \begin{array}{c}
u_{i j}=u_{i 0}-u_{j 0} \\
v_{i j}=v_{i 0}-v_{j 0} .
\end{array}
\end{gathered}
$$

Thus if we write $I_{D}(\boldsymbol{s}) \equiv I_{D}(\ell, m)$ and $\boldsymbol{a}_{k}(\boldsymbol{s}) \equiv \boldsymbol{a}_{k}(\ell, m)$, we can rewrite the dirty image (18) as

$$
\begin{aligned}
I_{D}(\boldsymbol{s})= & \sum_{i, j, k} V\left(u_{i j}\left(t_{k}\right), v_{i j}\left(t_{k}\right)\right) \\
& \cdot e^{2 \pi \jmath\left(u_{i 0}\left(t_{k}\right) \ell+v_{i 0}\left(t_{k}\right) m\right)} e^{-2 \pi \jmath\left(u_{j 0}\left(t_{k}\right) \ell+v_{j 0}\left(t_{k}\right) m\right)} \\
= & \sum_{i, j, k}\left(\boldsymbol{R}_{k}\right)_{i j}\left(\overline{\boldsymbol{a}}_{k}(\boldsymbol{s})\right)_{i}\left(\boldsymbol{a}_{k}(\boldsymbol{s})\right)_{j} \\
= & \sum_{k} \boldsymbol{a}_{k}^{\mathrm{H}}(\boldsymbol{s}) \boldsymbol{R}_{k} \boldsymbol{a}_{k}(\boldsymbol{s}) .
\end{aligned}
$$

(We omitted the optional weighting. Also note that, with noise, we have to replace $\boldsymbol{R}_{k}$ by $\boldsymbol{R}_{k}-\sigma^{2} \boldsymbol{I}$.) The iterative beam removing in CLEAN can now be posed as an iterative LS fitting between the sky model and the observed visibility [31]. Finding the brightest point $\boldsymbol{s}_{0}$ in the image is equivalent to trying to find a point source using classical Fourier beamforming, i.e.,

$$
\hat{\boldsymbol{s}}_{0}=\underset{\boldsymbol{s}}{\arg \max } \sum_{k=1}^{K} \boldsymbol{a}_{k}^{\mathrm{H}}(\boldsymbol{s})\left(\boldsymbol{R}_{k}-\sigma^{2} \boldsymbol{I}\right) \boldsymbol{a}_{k}(\boldsymbol{s}) .
$$

Thus the CLEAN algorithm can be regarded as a generalized classical sequential beamformer, where the brightest points are found one by one, and subsequently removed from $\boldsymbol{R}_{k}$ until the LS cost function (19) is minimized. An immediate consequence is that the estimated source locations will be biased: a well-known fact in array processing. When the sources are well separated, the bias is negligible compared to the standard deviation, otherwise it might be significant. This gives an explanation for the poor performance of the CLEAN in imaging extended structures (see, e.g., [26]). Another enhancement in the CLEAN can be made by turning it into an iterative scheme rather than sequential. In this case after estimating all point sources, we put one source at a time back into the data, and re-estimate it, using the estimates of the other point sources. This will improve the LS fit, and can easily be proved to converge. This approach is similar to the alternating projections approach for computing the deterministic ML DOA estimator [46].

\section{B. Minimum Variance Beamforming Approaches}

Once we view image formation/deconvolution as equivalent to DOA estimation with a moving array, we can try to adapt various other DOA estimators for handling the image formation. In particular, the deflation approach used in the CLEAN algorithm can be replaced by other source parameters estimators. One approach that seems particularly relevant in this context is the Minimum-Variance Distortionless Response (MVDR) method of beamforming [6]. The major new aspect here is the fact that the array is moving and that there are more sources than sensors.

Instead of working with the dirty image

$$
I_{D}(\boldsymbol{s})=\sum_{k} \boldsymbol{a}_{k}^{\mathrm{H}}(\boldsymbol{s}) \boldsymbol{R}_{k} a_{k}(\boldsymbol{s})
$$

the basis for high-resolution beamforming techniques is to look at more general "pseudo-spectra"

$$
I_{D}^{\prime}(\boldsymbol{s}):=\sum_{k} \boldsymbol{w}_{k}^{\mathrm{H}}(\boldsymbol{s}) \boldsymbol{R}_{k} \boldsymbol{w}_{k}(\boldsymbol{s})
$$

Here, $\boldsymbol{w}_{k}(\boldsymbol{s})$ is the beamformer pointing toward direction $\boldsymbol{s}$, and $I_{D}^{\prime}(\boldsymbol{s})$ is the output energy of that beamformer. Previously we had $\boldsymbol{w}_{k}(\boldsymbol{s})=\boldsymbol{a}_{k}(\boldsymbol{s})$; the objective is to construct beamformers that provide better separation of close sources.

A generalized MVDR follows by defining the problem as follows. At each time instance $k$ we would like to generate a weight vector $\boldsymbol{w}_{k}$ which minimizes the output power at time $k$ subject to the constraint that we have a fixed response toward the look direction $\boldsymbol{s}$ of the array, i.e.,

$$
\hat{\boldsymbol{w}}_{k}(\boldsymbol{s})=\underset{\boldsymbol{w}_{k}}{\operatorname{argmin}} \boldsymbol{w}_{k}^{\mathrm{H}} \hat{\boldsymbol{R}}_{\boldsymbol{k}} \boldsymbol{w}_{k} \quad \text { such that } \quad \boldsymbol{w}_{k}^{\mathrm{H}} \boldsymbol{a}_{k}(\boldsymbol{s})=1 .
$$

The solution to this problem is

$$
\hat{\boldsymbol{w}}_{k}=\beta_{k} \hat{\boldsymbol{R}}_{k}^{-1} \boldsymbol{a}_{k}(\boldsymbol{s}), \quad \text { where } \beta_{k}=\frac{1}{\boldsymbol{a}_{k}^{\mathrm{H}}(\boldsymbol{s}) \hat{\boldsymbol{R}}_{k}^{-1} \boldsymbol{a}_{k}(\boldsymbol{s})} .
$$

Inserting in (21) shows that the overall spectral estimator is given by

$$
I_{D}^{\prime}(\boldsymbol{s})=\sum_{k=1}^{K} \frac{1}{\boldsymbol{a}_{k}^{\mathrm{H}}(\boldsymbol{s}) \hat{\boldsymbol{R}}_{k}^{-1} \boldsymbol{a}_{k}(\boldsymbol{s})}
$$

and the locations of the strongest sources are given by the maxima of $I_{D}^{\prime}(\boldsymbol{s})$. It is known that the MVDR has improved resolution compared to the classical beamformer which is the basis for the CLEAN algorithm. Fig. 6 illustrates this by comparing a dirty image produced in the classical way to the dirty image corresponding to (23). In this simulation, we generated an extended structure by placing many point sources 


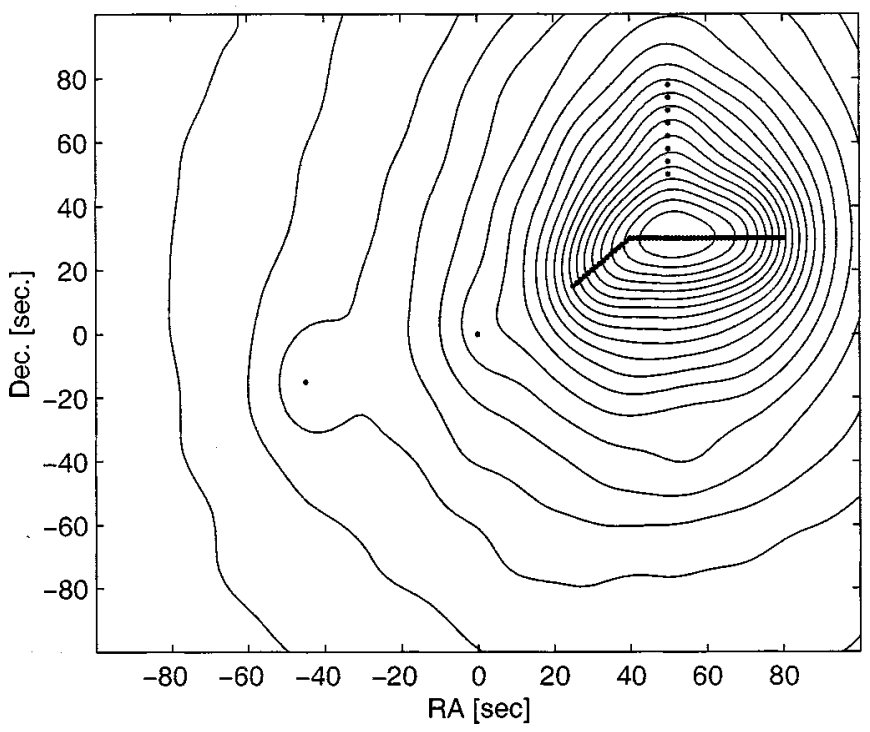

(a)

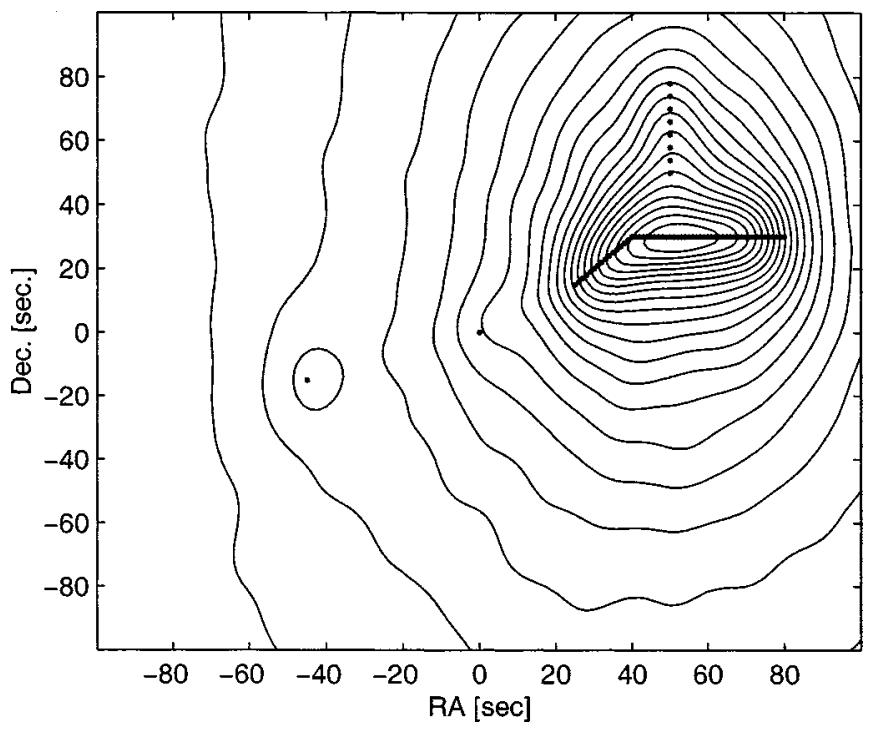

(b)

Fig. 6. (a) Conventional dirty image. (b) Dirty image using MVDR beamformimg. The dots represented the locations of the point sources modeled.

close to each other. The MVDR-based imaging produces a much sharper result.

Compared to CLEAN there is a slight computational loss. Another drawback of the MVDR is the fact that the noise distribution at the output of the beamformer is not identical toward all directions. Here we can use a modification [5] which demands that $\boldsymbol{w}_{k}$ have a functional form $\boldsymbol{w}_{k}=\beta_{k} \hat{\boldsymbol{R}}_{k}^{-1} \boldsymbol{a}_{k}(\boldsymbol{s})$, for some $\beta_{k}$, but now under the constraint that $\left\|\boldsymbol{w}_{k}\right\|^{2}=1$. This leads to the following estimator:

$$
I_{D}^{\prime \prime}(\boldsymbol{s})=\sum_{k=1}^{K} \frac{\boldsymbol{a}_{k}^{\mathrm{H}}(\boldsymbol{s}) \hat{\boldsymbol{R}}_{k}^{-1} \boldsymbol{a}_{k}(\boldsymbol{s})}{\boldsymbol{a}_{k}^{\mathrm{H}}(\boldsymbol{s}) \hat{\boldsymbol{R}}_{k}^{-2} \boldsymbol{a}_{k}(\boldsymbol{s})}, \quad \hat{\boldsymbol{s}}_{0}=\underset{\boldsymbol{s}}{\arg \max } I_{D}^{\prime \prime}(\boldsymbol{s}) .
$$

Many other techniques exist for estimating the point-source locations. A good overview of the various possibilities can be found in [16].

\section{CLEAN with Spatial Filtering}

Let us assume now that we have spatially filtered the covariance matrices $\hat{\boldsymbol{R}}_{k}$ by linear operations $L_{k}$, for example, projections. If we assume that all the interference is removed by the filtering, the measurement equation becomes

$$
\tilde{\boldsymbol{R}}_{k}:=\boldsymbol{L}_{k} \boldsymbol{R}_{k} \boldsymbol{L}_{k}^{\mathrm{H}}=\boldsymbol{L}_{k}\left[\boldsymbol{A}_{k}\left(\left\{\boldsymbol{s}_{l}\right\}\right) \boldsymbol{B} \boldsymbol{A}_{k}^{\mathrm{H}}\left(\left\{\boldsymbol{s}_{l}\right\}\right)+\sigma^{2} \boldsymbol{I}\right] \boldsymbol{L}_{k}^{\mathrm{H}} .
$$

This modifies the LS optimization problem to

$$
\begin{aligned}
& {\left[\left\{\hat{\boldsymbol{s}}_{\boldsymbol{l}}\right\}, \hat{\boldsymbol{B}}\right]=\underset{\left\{\boldsymbol{s}_{l}\right\}, \boldsymbol{B},\left\{\boldsymbol{\Gamma}_{k}\right\}}{\arg \min } \sum_{k=1}^{K}} \\
& \quad\left\|\boldsymbol{L}_{k}\left(\hat{\boldsymbol{R}}_{k}-\boldsymbol{A}_{k}\left(\left\{\boldsymbol{s}_{l}\right\}\right) \boldsymbol{B} \boldsymbol{A}_{k}^{\mathrm{H}}\left(\left\{\boldsymbol{s}_{l}\right\}\right)-\sigma^{2} \boldsymbol{I}\right) \boldsymbol{L}_{k}^{\mathrm{H}}\right\|_{F} .
\end{aligned}
$$

The cost function is similar to (29) and thus its minimization does not pose stronger computational demands. Indeed, as we mentioned before in Section V-B, if we follow the classical Fourier-type imaging we end up with a deconvolution problem with a space-varying beam, but the CLEAN algorithm is simply extended to take this into account. Here, we develop the extension more carefully, taking note of the fact that the noise structure after projections is not white anymore.

In the case of spatially filtered signals the classical beamformer follows from the previous by replacing $\boldsymbol{a}_{k}(\boldsymbol{s})$ by the effective array response $\boldsymbol{L}_{k} \boldsymbol{a}_{k}(\boldsymbol{s})$, i.e.,

$$
\begin{aligned}
I_{D}^{\prime}(\boldsymbol{s}) & =\sum_{k=1}^{K} \boldsymbol{a}_{k}^{\mathrm{H}}(\boldsymbol{s}) \boldsymbol{L}_{k}^{\mathrm{H}}\left(\boldsymbol{L}_{k} \hat{\boldsymbol{R}}_{k} L_{k}-\sigma^{2} \boldsymbol{L}_{k} \boldsymbol{L}_{k}^{\mathrm{H}}\right) \boldsymbol{L}_{k} \boldsymbol{a}_{k}(\boldsymbol{s}) \\
& =\sum_{k=1}^{K} \boldsymbol{a}_{k}^{\mathrm{H}}(\boldsymbol{s})\left(\boldsymbol{L}_{k}^{\mathrm{H}} \boldsymbol{L}_{k} \hat{\boldsymbol{R}}_{k} \boldsymbol{L}_{k}^{\mathrm{H}} \boldsymbol{L}_{k}-\sigma^{2} \boldsymbol{L}_{k}^{\mathrm{H}} \boldsymbol{L}_{k} \boldsymbol{L}_{k}^{\mathrm{H}} \boldsymbol{L}_{k}\right) \boldsymbol{a}_{k}(\boldsymbol{s}) \\
& =\sum_{k=1}^{K} \boldsymbol{a}_{k}^{\mathrm{H}}(\boldsymbol{s}) \hat{\boldsymbol{R}}_{k}^{\prime} \boldsymbol{a}_{k}(\boldsymbol{s})
\end{aligned}
$$

where

$$
\hat{\boldsymbol{R}}_{k}^{\prime}=\boldsymbol{L}_{k}^{\mathrm{H}} \boldsymbol{L}_{k} \hat{\boldsymbol{R}}_{k} \boldsymbol{L}_{k}^{\mathrm{H}} \boldsymbol{L}_{k}-\sigma^{2} \boldsymbol{L}_{k}^{\mathrm{H}} \boldsymbol{L}_{k} \boldsymbol{L}_{k}^{\mathrm{H}} \boldsymbol{L}_{k} .
$$

Therefore, the step of finding the brightest point $\boldsymbol{s}_{0}$ in the image can be implemented using the fast Fourier transform (FFT) in the same way it is implemented in the CLEAN algorithm, but acting on $\hat{\boldsymbol{R}}_{k}^{\prime}$ instead of the original visibilities. Similarly, the contribution of a source at location $s_{0}$ in a single covariance matrix $\tilde{\boldsymbol{R}}_{k}$ is a multiple of $L_{k} \boldsymbol{a}_{k}\left(\boldsymbol{s}_{0}\right) \boldsymbol{a}_{k}^{\mathrm{H}}\left(\boldsymbol{s}_{0}\right) \boldsymbol{L}_{k}^{\mathrm{H}}$, and hence the response in the dirty image $I_{D}^{\prime}(\boldsymbol{s})$ is given by

$$
B\left(\boldsymbol{s}, \boldsymbol{s}_{0}\right):=\sum_{k=1}^{K} \boldsymbol{a}_{k}^{\mathrm{H}}(\boldsymbol{s}) \boldsymbol{L}_{k}^{\mathrm{H}}\left(\boldsymbol{L}_{k} \boldsymbol{a}_{k}\left(\boldsymbol{s}_{0}\right) \boldsymbol{a}_{k}^{\mathrm{H}}\left(\boldsymbol{s}_{0}\right) \boldsymbol{L}_{k}^{\mathrm{H}}\right) \boldsymbol{L}_{k} \boldsymbol{a}_{k}(\boldsymbol{s}) .
$$

This is the space-varying beam. The extended CLEAN algorithm after spatial filtering now follows immediately and is given in Table II.

To test the algorithm, we have taken an array configuration with $p=14$ telescopes as in WSRT, and generated four equalpowered point sources centered around right ascension $32^{\circ}$ and declination $60^{\circ}$, with a signal-to-noise ratio of $-20 \mathrm{~dB}$ for each of the sources. To simulate the effect of spatial filtering, we placed an interferer at a fixed terrestrial location (hence varying 
TABLE II

The Clean Algorithm with Spatial Filtering

Compute $\hat{\mathbf{R}}^{\prime}$ using (27)
$I_{D}^{\prime}(\mathbf{s})=\sum_{k=1}^{K} \mathbf{a}_{k}^{\mathrm{H}}(\mathbf{s}) \hat{\mathbf{R}}_{k}^{\prime} \mathbf{a}_{k}(\mathbf{s})$
$l=0$
while $I_{D}^{\prime}$ is not noise-like:
\[ \left[\begin{array}{l}\mathbf{s}_{l}=\arg \max I_{D}^{\prime}(\mathbf{s}) \\ \text { Compute } B\left(\mathbf{s}, \mathbf{s}_{l}\right) \text { using }(28) \\ \lambda_{l}=I_{D}\left(\mathbf{s}_{l}\right) / B\left(\mathbf{s}_{l}, \mathbf{s}_{l}\right) \\ I_{D}^{\prime}(\mathbf{s}):=I_{D}^{\prime}(\mathbf{s})-\gamma \lambda_{l} B\left(\mathbf{s}, \mathbf{s}_{l}\right) \\ l=\end{array}\right. \]
$I=I_{D}^{\prime}+\sum_{l} \gamma \lambda_{l} B_{\text {synth }}\left(\mathbf{s}-\mathbf{s}_{l}\right)$

compared to the look direction of the array), and with INR $=5$ dB. $K=100$ sample covariance matrices $\hat{\boldsymbol{R}}_{k}$ were generated, uniformly spread along $12 \mathrm{~h}$, and each based on $N=1000 \mathrm{sam}$ ples. Fig. 7(a)-(c) shows the dirty image without interference present, the effect of the interferer on the dirty image, and the dirty image after estimating and removing the interferer using spatial projections. Clearly, with interference present but not removed, the sources are completely masked out (note the change in scale between the first two images). After estimating and projecting out the interferer, in the third image, we obtain nominally the same image as in the interference-free case, but the sidelobe patterns are different (as we demonstrated before, they are, in fact, space-varying). The circles in the third image mark the true location of the point sources, and the + symbols mark the locations estimated by the extended CLEAN algorithm of Table II. We can clearly see that the correct locations have been obtained. This would not be the case with the unmodified CLEAN algorithm.

\section{Self-Calibration}

To finish this section, we consider the situation where also the array gains $\boldsymbol{\Gamma}_{k}$ are unknown. In this case, the model fitting equation without spatial filtering, (19), generalizes to

$$
\begin{aligned}
& {\left[\left\{\hat{\boldsymbol{s}}_{l}\right\}, \hat{\boldsymbol{B}},\left\{\hat{\boldsymbol{\Gamma}}_{k}\right\}\right]=\underset{\left\{\boldsymbol{s}_{l}\right\}, \boldsymbol{B},\left\{\boldsymbol{\Gamma}_{k}\right\}}{\operatorname{argmin}} \sum_{k=1}^{K}} \\
& \quad\left\|\hat{\boldsymbol{R}}_{k}-\boldsymbol{\Gamma}_{k} \boldsymbol{A}_{k}\left(\left\{\boldsymbol{s}_{l}\right\}\right) \boldsymbol{B} \boldsymbol{A}_{k}^{\mathrm{H}}\left(\left\{\boldsymbol{s}_{l}\right\}\right) \boldsymbol{\Gamma}_{k}^{\mathrm{H}}-\sigma^{2} \boldsymbol{I}\right\|_{F} .
\end{aligned}
$$

The solution can be obtained by the "self-cal" algorithm [25]: an alternating LS algorithm which solves iteratively for the parameters $\boldsymbol{B},\left\{\boldsymbol{s}_{l}\right\}$ by a CLEAN step (with fixed gains), and the gain parameters $\left\{\boldsymbol{\Gamma}_{k}\right\}$ by a calibration step (with fixed source parameters $\boldsymbol{B},\left\{\boldsymbol{s}_{\boldsymbol{l}}\right\}$ ).

It has not been noted before in the literature that the latter step admits a direct algebraic solution. Indeed, to minimize (29) with fixed $\left\{\boldsymbol{A}_{\boldsymbol{k}}\right\}$ and $\boldsymbol{B}$, we can minimize separately for each $k$ the related expression

$$
\min _{\left\{\boldsymbol{\Gamma}_{k}\right\}}\left\|\hat{\boldsymbol{R}}_{k}-\boldsymbol{\Gamma}_{k} \boldsymbol{A}_{k} \boldsymbol{B} \boldsymbol{A}_{k}^{\mathrm{H}} \boldsymbol{\Gamma}_{k}^{\mathrm{H}}-\sigma^{2} \boldsymbol{I}\right\|_{F} .
$$

Let $\boldsymbol{g}_{k}$ be the vector of diagonal elements $\gamma_{k, i}$ of $\boldsymbol{\Gamma}_{k}$. Given an estimate of $\boldsymbol{A}_{k}$ and $\boldsymbol{B}$ we can define for each $k$ a $(p \times p)$ matrix $\hat{\boldsymbol{X}}_{k}$ with entries

$$
\left(\hat{\boldsymbol{X}}_{k}\right)_{i j}=\frac{\left(\hat{\boldsymbol{R}}_{k}-\sigma^{2} \boldsymbol{I}\right)_{i j}}{\left(\boldsymbol{A}_{k} B A_{k}^{\mathrm{H}}\right)_{i j}}
$$

and fit $\boldsymbol{g}_{k}$ with entries $g_{k, i}$ such that $\left(\hat{\boldsymbol{X}}_{k}\right)_{i j}=g_{k, i} \bar{g}_{k, j}$. In the usual self-calibration algorithm, this equation is solved iteratively for all two-by-two submatrices of $\hat{\boldsymbol{X}}_{k}$ using the so-called gain and phase closure relations. Instead, we note here that the problem admits a more elegant solution, since in matrix form, we have

$$
\hat{\boldsymbol{X}}_{k}=\boldsymbol{g}_{k} \boldsymbol{g}_{k}^{\mathrm{H}} .
$$

This asks for the best Hermitian rank-one approximation to the matrix $\boldsymbol{X}_{k}$, which is known to be given by $\hat{\boldsymbol{g}}_{k}=\sqrt{\lambda_{1}} \boldsymbol{v}_{1}$ where $\lambda_{1}$ is the largest eigenvalue of $\hat{\boldsymbol{X}}_{k}$ and $\boldsymbol{v}_{1}$ its corresponding eigenvector.

More work is needed to generalize this to the model equation with spatial filtering. With fixed $A_{k}$ and $B$, the minimization problem for the gain parameters is

$$
\min _{\left\{\boldsymbol{\Gamma}_{k}\right\}}\left\|\boldsymbol{L}_{k}\left(\hat{\boldsymbol{R}}_{k}-\boldsymbol{\Gamma}_{k} \boldsymbol{A}_{k} \boldsymbol{B} \boldsymbol{A}_{k}^{\mathrm{H}} \boldsymbol{\Gamma}_{k}^{\mathrm{H}}-\sigma^{2} \boldsymbol{I}\right) \boldsymbol{L}_{k}^{\mathrm{H}}\right\|_{F} \cdot
$$

Let $\operatorname{vec}(\cdot)$ denote the operation of stacking all columns of a matrix into a vector, and let $\otimes$ denote the Kronecker product. A general property valid for matrices of compatible size is

$$
\operatorname{vec}(A B C)=\left(C^{T} \otimes A\right) \operatorname{vec}(B) .
$$




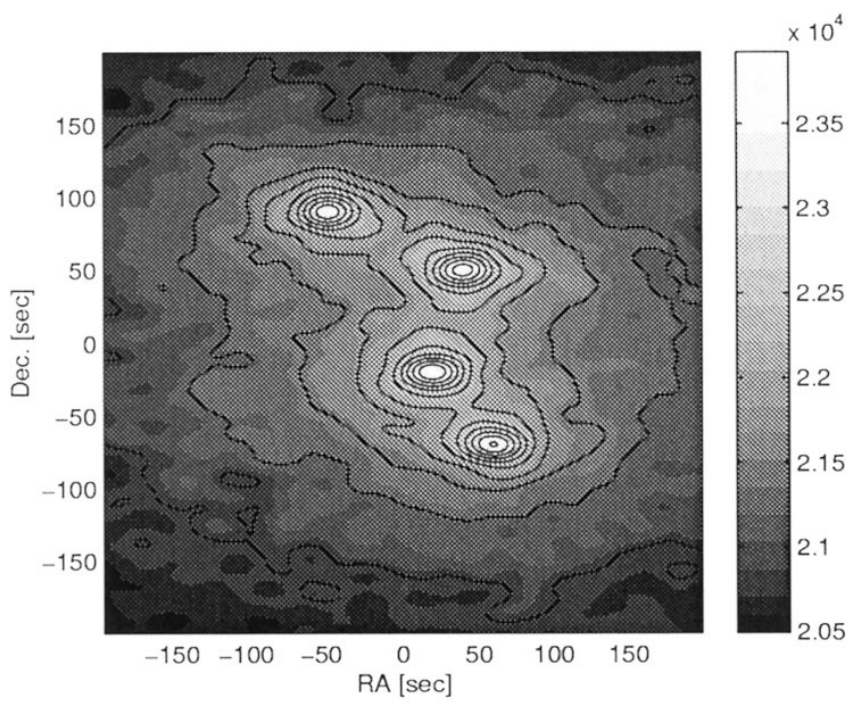

(a)

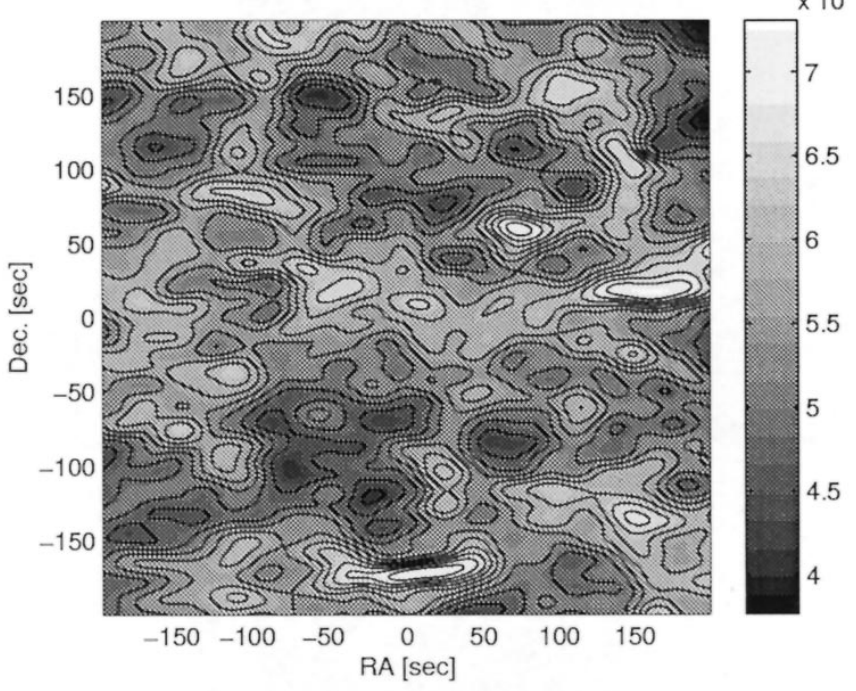

(b)

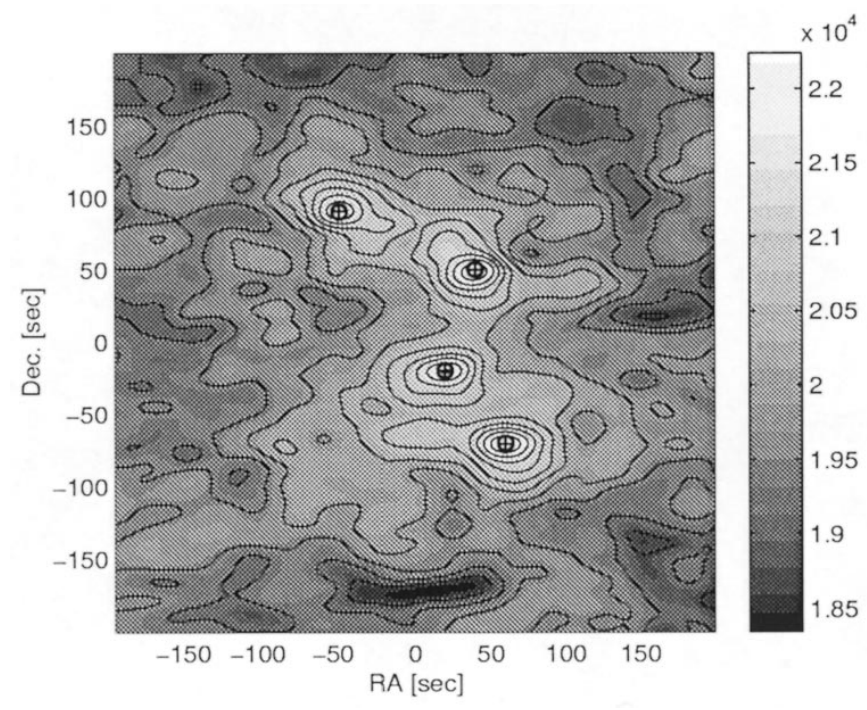

(c)

Fig. 7. Dirty images of four point sources. (a) No interference. (b) Unsuppressed interference (INR $=5 \mathrm{~dB}$ ). (c) After spatial filtering.
Application to the present context gives that (31) asks for the solution in least squares sense of

$$
\begin{aligned}
& \left(\overline{\boldsymbol{L}}_{k} \otimes \boldsymbol{L}_{k}\right) \operatorname{vec}\left(\hat{\boldsymbol{R}}_{k}-\sigma^{2} \boldsymbol{I}\right) \\
& \quad=\left(\overline{\boldsymbol{L}}_{k} \otimes \boldsymbol{L}_{k}\right) \operatorname{vec}\left(\boldsymbol{\Gamma}_{k} \boldsymbol{A}_{k} \boldsymbol{B} \boldsymbol{A}_{k}^{\mathrm{H}} \boldsymbol{\Gamma}_{k}^{\mathrm{H}}\right) \\
& \quad=\left(\overline{\boldsymbol{L}}_{k} \otimes \boldsymbol{L}_{k}\right) \operatorname{diag}\left(\operatorname{vec}\left(\boldsymbol{A}_{k} \boldsymbol{B} \boldsymbol{A}_{k}^{\mathrm{H}}\right)\right)\left(\overline{\boldsymbol{g}}_{k} \otimes \boldsymbol{g}_{k}\right)
\end{aligned}
$$

which has the form

$$
\boldsymbol{h}_{k}=\boldsymbol{F}_{k} x_{k}, \quad \boldsymbol{x}_{k}=\overline{\boldsymbol{g}}_{k} \otimes \boldsymbol{g}_{k} .
$$

If $\boldsymbol{F}_{k}$ has a left inverse $\boldsymbol{F}_{k}^{\dagger}$, we can find a unique LS solution for $\boldsymbol{x}_{k}$, i.e., $\hat{\boldsymbol{x}}_{k}=\boldsymbol{F}_{k}^{\dagger} \boldsymbol{h}_{k}$, and then fit $\hat{\boldsymbol{x}}_{k}=\overline{\boldsymbol{g}}_{k} \otimes \boldsymbol{g}_{k}$, or equivalently $\hat{\boldsymbol{X}}_{k}=\boldsymbol{g}_{k} \boldsymbol{g}_{k}^{\mathrm{H}}$, where $\hat{\boldsymbol{X}}_{k}$ is derived from $\hat{\boldsymbol{x}}_{k}$ by unstacking, i.e., $\operatorname{vec}\left(\hat{\boldsymbol{X}}_{k}\right)=\hat{\boldsymbol{x}}_{k}$. This is precisely the solution which we obtained before.

However, in the present case $L_{k}$ is a projection operator and hence $\boldsymbol{F}_{k}$ is not invertible. It is easy to see from examples, such as taking $L_{k}=\left[\begin{array}{l}I 0 \\ 00\end{array}\right]$, that in these cases $\boldsymbol{\Gamma}_{k}$ is not identifiable. A solution can be obtained if we make the reasonable assumption that $\boldsymbol{\Gamma}_{k}$ is constant over several epochs, say for $k, k+1, \cdots, k+$ $M-1$, and that $\boldsymbol{L}_{k}$ is sufficiently varying over this period, for example, due to multipath or fringe corrections. In that case, we obtain

$$
\left[\begin{array}{l}
\boldsymbol{h}_{k} \\
\boldsymbol{h}_{k+1} \\
\vdots \\
\boldsymbol{h}_{k+M-1}
\end{array}\right]=\left[\begin{array}{l}
\boldsymbol{F}_{k} \\
\boldsymbol{F}_{k+1} \\
\vdots \\
\boldsymbol{F}_{k+M-1}
\end{array}\right] \boldsymbol{x}_{k}, \quad \boldsymbol{x}_{k}=\bar{g}_{k} \otimes \boldsymbol{g}_{k} .
$$

For sufficiently large $M$, the block matrix is tall and of full column rank, and has a left inverse. We can thus solve for an unstructured LS estimate $\hat{\boldsymbol{x}}_{k}$, and subsequently fit $\hat{\boldsymbol{x}}_{k}=\overline{\boldsymbol{g}}_{k} \otimes \boldsymbol{g}_{k}$.

The minimal number $M$ of linearly independent matrices $L_{k}$ which are needed follows from counting dimensions. If we project out $q$ interferers on $p$ antennas, $\boldsymbol{L}_{k}$ has $p-q$ independent rows and $p$ columns. Hence $\boldsymbol{F}_{k}$ has $(p-q)^{2}$ independent rows and $p^{2}$ columns, and we need $M(p-q)^{2} \geq p^{2}$. This gives modest requirements: if for $p=14$ we take $M=2$, then we can accept up to $q=4$ interferers; with $M=4$, up to $q=7$.

In summary, we have obtained an elegant and computationally nonintensive extension of the "self-cal" algorithm to complement the space-filtering CLEAN algorithm.

\section{MAXIMUM-LIKELIHOOD IMAGING}

\section{A. Maximum-Likelihood Functional}

Let us consider the imaging step from a more fundamental viewpoint. In principle, the construction of the image using the observed correlation matrices and assuming the parametric model can be viewed as a parameter estimation problem. One of the most important inference methods is the maximum likelihood (ML) method. Given a parametric family of probabilistic models for the received data, choose the parameters that maximize the probability of obtaining the observed data. 
This is different than the most probable image approach [8] where no parametric model is imposed on the image, leading to maximum entropy image formation. Maximum-likelihood estimators (MLE's) are known to be consistent and asymptotically statistically efficient (i.e., they provide unbiased estimators with minimum variance) under very general conditions, and thus are the natural choice for many parameter estimation problems.

In deriving the MLE of the image parameters, we need a parametric family of models for the astronomical signals. A reasonable assumption regarding the astronomical data is Gaussianity of the temporal samples. ${ }^{6}$ This assumption is used in current imaging systems which rely only on second-order statistics (both temporal and spatial). For simplicity we further assume that the samples are temporally white (valid for the relatively narrow bands processed, while over very large bands the blackbody radiation pattern should be taken into account). For further discussion on emission mechanisms, and the resulting physical models of emission the reader is referred to [28]. Contrary to the claim in [37], the corresponding MLE is not equivalent to parametric optimization of the CLEAN cost function. Using the discrete point-source model we obtain

$$
\boldsymbol{R}_{k}=\boldsymbol{\Gamma}_{k} \boldsymbol{A}_{k}\left(\left\{\boldsymbol{s}_{l}\right\}\right) \boldsymbol{B} A_{k}^{\mathrm{H}}\left(\left\{\boldsymbol{s}_{l}\right\}\right) \boldsymbol{\Gamma}_{k}^{\mathrm{H}}+\sigma^{2} \boldsymbol{I}
$$

where the $d$ astronomical sources are Gaussian with covariance matrix $\boldsymbol{B}=\operatorname{diag}\left[I_{1}, \cdots, I_{d}\right]$ and sky coordinates $\left\{\boldsymbol{s}_{l}\right\}_{l=1}^{d}$, and the noise is Gaussian with covariance $\sigma^{2} \boldsymbol{I}$. Let $\hat{\boldsymbol{R}}_{k}$ be the sample covariance matrix during the $k$ th epoch, based on $N_{k}$ collected samples. The likelihood of the observations at the $k$ th epoch given map parameters $\left\{\boldsymbol{s}_{l}\right\}, \boldsymbol{B}, \sigma^{2}, \boldsymbol{\Gamma}_{k}$ is then given by [2]

$$
p\left(\hat{\boldsymbol{R}}_{k} \mid\left\{\boldsymbol{s}_{l}\right\}, \boldsymbol{B}, \sigma^{2}, \boldsymbol{\Gamma}_{k}\right)=\left(\frac{1}{\pi^{p}\left|\boldsymbol{R}_{k}\right|} e^{-\operatorname{tr}\left(\boldsymbol{R}_{k}^{-1} \hat{\boldsymbol{R}}_{k}\right)}\right)^{N_{k}} .
$$

Using all $K$ observation epochs we obtain that the log-likelihood function is given by (after omitting constants)

$$
\begin{aligned}
\mathcal{L}\left(\hat{\boldsymbol{R}}_{1}, \cdots, \hat{\boldsymbol{R}}_{K} \mid \boldsymbol{B},\left\{\boldsymbol{s}_{l}\right\},\left\{\boldsymbol{\Gamma}_{k}\right\}, \sigma^{2}\right) & \\
= & -\sum_{k=1}^{K} N_{k} \log \left|\boldsymbol{R}_{k}\right|-\sum_{k=1}^{K} N_{k} \operatorname{tr}\left(\boldsymbol{R}_{k}^{-1} \hat{\boldsymbol{R}}_{k}\right) .
\end{aligned}
$$

The MLE is found by maximizing (34) over $\boldsymbol{B},\left\{\boldsymbol{s}_{l}\right\},\left\{\boldsymbol{\Gamma}_{k}\right\}, \sigma^{2}$. This maximization problem is prohibitively complex and hence some simplifications are needed. In some simplified cases in DOA estimation this has been dealt with. The Gaussian signals model for a static array with perfect calibration have been considered by [4] which eliminated analytically some of the parameters. Derivation of the MLE for a single source in white Gaussian noise for the simplified model appeared in [45].

Since the CLEAN gives an approximate solution to the deconvolution problem we can use it to initialize an ML search. In this case, the CLEAN components serve as initial estimates to the MLE and the MLE serves the purpose of fine focusing of the image, by shifting each point source to its true value. The ML search itself can be done either using a gradient search, a Newton search based on the Fisher information matrix, or an EM algorithm. In Appendix B we present the expressions of the

\footnotetext{
${ }^{6}$ The assumption is valid for continuum emission, while for spectral line observations certain adjustments of the model are needed to include the lines structure.
}

gradient and the Hessian needed for the optimization. Since a good initialization is very important for optimizing the complicated equation (34) we will continue to derive an approximate coordinate descent MLE which is computationally simpler, and show its connection to CLEAN.

\section{B. Single Source in Colored Noise}

One simplified approach to solve the MLE (34) which leads to good results in LS problems is the deflation approach or coordinate descent algorithm. In this approach, the sources are extracted one by one and once we have obtained estimates of parameters of all sources we iterate the optimization along each parameter fixing the other parameters. Some examples of this approach are the alternating projections algorithm [46] and the estimation of multipath parameters [41].

Our basic approach to remove the effect of previously estimated sources will be to lump their contribution into the "noise part" of the covariance matrix. This essentially provides an $a$ posteriori estimate of the noise covariance matrix, before continuing to estimate the next source. Hence an important component of the algorithm will be the ML estimation of a single source in colored noise with known covariance matrix. In what follows we will derive an approximate ML estimator for this specific case. In order to reduce the notational complexity we will restrict ourselves to perfectly calibrated arrays, i.e., $\boldsymbol{\Gamma}_{k}=\boldsymbol{I}$ for all $k$, and note that estimating the calibration parameters will be done in a separate stage as it is currently done in the self-calibration scheme.

In order to reduce computational complexity even further, we would like to perform the source power estimation (conditioned on the location parameter) analytically. This will result in a two-dimensional search over the field of view for the location with highest likelihood for a point source, a task of moderate complexity.

Assume that we are given a set of covariance matrices $\left\{\hat{\boldsymbol{R}}_{k}: k=1, \cdots, K\right\}$ which are sample estimates of $\boldsymbol{R}_{k}$ where

$$
\boldsymbol{R}_{k}=\lambda \boldsymbol{a}_{k}(\boldsymbol{s}) \boldsymbol{a}_{k}^{\mathrm{H}}(\boldsymbol{s})+\boldsymbol{C}_{k}
$$

and $\boldsymbol{C}_{k}$ is the noise covariance matrix (assumed to be known).

The log-likelihood function is given by

$$
\mathcal{L}\left(\hat{\boldsymbol{R}}_{1}, \cdots, \hat{\boldsymbol{R}}_{K} \mid \lambda, \boldsymbol{s}\right)=-\sum_{k=1}^{K} N_{k}\left[\log \left|\boldsymbol{R}_{k}\right|+\operatorname{tr}\left(\boldsymbol{R}_{k}^{-1} \hat{\boldsymbol{R}}_{k}\right)\right] .
$$

Substituting (35) into (36) we obtain

$$
\begin{aligned}
\mathcal{L}\left(\hat{\boldsymbol{R}}_{1}, \cdots, \hat{\boldsymbol{R}}_{K} \mid \lambda, \boldsymbol{s}\right)=- & \sum_{k=1}^{K} N_{k}\left[\log \left|\lambda \boldsymbol{a}_{k}(\boldsymbol{s}) \boldsymbol{a}_{k}^{\mathrm{H}}(\boldsymbol{s})+\boldsymbol{C}_{k}\right|\right. \\
& \left.+\operatorname{tr}\left(\left(\lambda \boldsymbol{a}_{k}(\boldsymbol{s}) \boldsymbol{a}_{k}^{\mathrm{H}}(\boldsymbol{s})+\boldsymbol{C}_{k}\right)^{-1} \hat{\boldsymbol{R}}_{k}\right)\right] .
\end{aligned}
$$

Further manipulation using $\operatorname{tr}(\boldsymbol{A B})=\operatorname{tr}(B A)$ and $|A B|=$ $|\boldsymbol{A}||\boldsymbol{B}|$ yields

$$
\begin{aligned}
\mathcal{L}\left(\hat{\boldsymbol{R}}_{1}, \cdots, \hat{\boldsymbol{R}}_{K} \mid \lambda, \boldsymbol{s}\right) & \\
=-\sum_{k=1}^{K} N_{k}[ & \log \left|\lambda \boldsymbol{C}^{-1} \boldsymbol{a}_{k}(\boldsymbol{s}) \boldsymbol{a}_{k}^{\mathrm{H}}(\boldsymbol{s})+\boldsymbol{I}\right|+\log \left|C_{k}\right| \\
& \left.+\operatorname{tr}\left(\left(\lambda \boldsymbol{C}_{k}^{-1} \boldsymbol{a}_{k}(\boldsymbol{s}) \boldsymbol{a}_{k}^{\mathrm{H}}(\boldsymbol{s})+\boldsymbol{I}\right)^{-1} \boldsymbol{C}_{k}^{-1} \hat{\boldsymbol{R}}_{k}\right)\right] .
\end{aligned}
$$


Since $\log \left|\boldsymbol{C}_{k}\right|$ does not depend on the parameters, the MLE is given by minimizing

$$
\begin{aligned}
& \mathcal{L}^{\prime}\left(\hat{\boldsymbol{R}}_{1}, \cdots, \hat{\boldsymbol{R}}_{K} \mid \lambda, \boldsymbol{s}\right) \\
& =\sum_{k=1}^{K} N_{k}\left[\log \left|\lambda \boldsymbol{C}_{k}^{-1} \boldsymbol{a}_{k}(\boldsymbol{s}) \boldsymbol{a}_{k}^{\mathrm{H}}(\boldsymbol{s})+I\right|\right. \\
& \left.+\operatorname{tr}\left(\left(\lambda \boldsymbol{C}_{k}^{-1} \boldsymbol{a}_{k}(\boldsymbol{s}) \boldsymbol{a}_{k}^{\mathrm{H}}(\boldsymbol{s})+\boldsymbol{I}\right) \boldsymbol{C}_{k}^{-1} \hat{\boldsymbol{R}}_{k}\right)\right]
\end{aligned}
$$

After some algebraic manipulations described in the Appendix, we obtain that we have to minimize

$$
\begin{aligned}
& \mathcal{L}^{\prime \prime}\left(\hat{\boldsymbol{R}}_{1}, \cdots, \hat{\boldsymbol{R}}_{K} \mid \lambda, \boldsymbol{s}\right) \\
&=\sum_{k=1}^{K} N_{k}\left[\log \left(1+\lambda \alpha_{k}\right)-\frac{\lambda \beta_{k}}{1+\lambda \alpha_{k}}\right]
\end{aligned}
$$

where

$$
\alpha_{k}=\boldsymbol{a}_{k}^{\mathrm{H}}(\boldsymbol{s}) \boldsymbol{C}_{k}^{-1} \boldsymbol{a}_{k}(\boldsymbol{s})
$$

and

$$
\beta_{k}=\boldsymbol{a}_{k}^{\mathrm{H}}(\boldsymbol{s}) \boldsymbol{C}_{k}^{-1} \hat{\boldsymbol{R}}_{k} \boldsymbol{C}_{k}^{-1} \boldsymbol{a}_{k}(\boldsymbol{s}) .
$$

Taking the derivative of the left-hand side with respect to $\lambda$ yields that the MLE of $\lambda$ is given by solving the equation

$$
\frac{\partial \mathcal{L}^{\prime \prime}}{\partial \lambda}=\sum_{k=1}^{K} N_{k}\left[\frac{\alpha_{k}}{1+\lambda \alpha_{k}}-\frac{\beta \beta_{k}}{1+\lambda \alpha_{k}}+\frac{\lambda \alpha_{k} \beta_{k}}{\left(1+\lambda \alpha_{k}\right)^{2}}\right]=0 .
$$

Equation (38) is highly nonlinear and hard to solve in the general case. We propose to simplify the problem by the following procedure. First estimate the source power using each of the covariance matrices separately and then average the estimates. Assuming that the ML estimate is consistent the above estimate will still be consistent. If we further assume that the statistical behavior of the various estimates is approximately the same (i.e., the Fisher information based on each time observation is almost constant) then the estimator is still efficient. This is formulated in the following lemma.

Lemma VII.1: Assume that

$$
\mathcal{L}(\boldsymbol{x} \mid \lambda)=\sum_{k=1}^{K} \mathcal{L}_{k}\left(x_{k} \mid \lambda\right)
$$

Let $\hat{\lambda}_{k}=\arg \max _{\lambda} \mathcal{L}_{k}\left(\boldsymbol{x}_{k} \mid \lambda\right)$ be the MLE based on the $k$ th block of data. Assume that all $J_{k}$ are equal, say $J_{k}(\lambda)=J$, where $J_{k}$ is the Fisher information for $\lambda_{k}$ based on the data $x_{k}$, and the likelihood $\mathcal{L}_{k}\left(\boldsymbol{x}_{k} \mid \lambda\right)$. Then $\bar{\lambda}=(1 / K) \sum_{k=1}^{K} \hat{\lambda}_{k}$ is an asymptotically efficient estimator of $\lambda$.

Proof: The Fisher information of the overall likelihood is given by $\sum_{k=1}^{K} J_{k}$. Thus the CRB on estimating $\lambda$ is given by

$$
\operatorname{var}(\hat{\lambda}) \geq \frac{1}{\sum_{k=1}^{K} J_{k}}=\frac{1}{K J}
$$

Since the MLE is asymptotically efficient (in the total number of samples $\sum_{k}, N_{k}$ ) its asymptotic variance achieves the CRB. On the other hand, if we estimate $\lambda$ based on the $k$ th block we obtain (by its asymptotic efficiency in $N_{k}$ ) that it has a variance given by

$$
\operatorname{var}\left(\hat{\lambda}_{k}\right)=\frac{1}{J_{k}}
$$

Now combining the various estimates we obtain

$$
\operatorname{var}(\bar{\lambda})=\frac{1}{K J}
$$

By the inequality of the harmonic and arithmetic mean we know that if the Fisher information is different at some time instances, then the averaged estimator has a larger variance, but the difference depends on the variation of the $J_{k}$ 's. However, this degradation in performance gives us a large computational saving. By the additivity of the derivative we obtain from (38) that for each $k$ the MLE $\hat{\lambda}_{k}$ based on $\hat{\boldsymbol{R}}_{k}$ is given by

$$
\hat{\lambda}_{k}=\frac{\boldsymbol{a}_{k}^{\mathrm{H}}(\boldsymbol{s})\left(\boldsymbol{C}_{k}^{-1} \hat{\boldsymbol{R}}_{k} C_{k}^{-1}-C_{k}^{-1}\right) \boldsymbol{a}_{k}(\boldsymbol{s})}{\left(\boldsymbol{a}_{k}^{\mathrm{H}}(\boldsymbol{s}) C_{k}^{-1} \boldsymbol{a}_{k}(\boldsymbol{s})\right)^{2}} .
$$

Equivalently, this can be written as

$$
\hat{\lambda}_{k}=\frac{\boldsymbol{a}_{k}^{\mathrm{H}}(\boldsymbol{s}) \boldsymbol{C}_{k}^{-1}\left(\hat{\boldsymbol{R}}_{k}-\boldsymbol{C}_{k}\right) \boldsymbol{C}_{k}^{-1} \boldsymbol{a}_{k}(\boldsymbol{s})}{\left(\boldsymbol{a}_{k}^{\mathrm{H}}(\boldsymbol{s}) C_{k}^{-1} a_{k}(\boldsymbol{s})\right)^{2}}
$$

and hence

$$
\bar{\lambda}=\frac{1}{\sum_{k=1}^{K} N_{k}} \sum_{k=1}^{K} N_{k}\left[\frac{a_{k}^{\mathrm{H}}(\boldsymbol{s}) \boldsymbol{C}_{k}^{-1}\left(\hat{\boldsymbol{R}}_{k}-C_{k}\right) \boldsymbol{C}_{k}^{-1} a_{k}(\boldsymbol{s})}{\left(\boldsymbol{a}_{k}^{\mathrm{H}}(\boldsymbol{s}) \boldsymbol{C}_{k}^{-1} \boldsymbol{a}_{k}(\boldsymbol{s})\right)^{2}}\right] .
$$

Now that we have this approximate ML (AML) estimate of $\lambda$ we can plug it into the likelihood function and obtain that we have to maximize over the field of view the following:

$$
\hat{\boldsymbol{s}}=\underset{\boldsymbol{s}}{\arg \max } \sum_{k=1}^{K} \log \left(\left|1+\bar{\lambda}(\boldsymbol{s}) \alpha_{k}(s)\right|\right)-\frac{\bar{\lambda}(\boldsymbol{s}) \beta_{k}(\boldsymbol{s})}{1+\bar{\lambda}(\boldsymbol{s}) \alpha_{k}(\boldsymbol{s})}
$$

The proposed coordinate-wise AML estimator is now summarized in Table III. ${ }^{7}, 8$

Alternatively to substituting back into the likelihood we can try to find the direction which maximizes the received power. It is interesting to observe that the CLEAN algorithm can be derived as an approximation to this power maximizing estimator. To that end maximize the power received from direction $\boldsymbol{s}$, i.e.,

$$
\hat{\boldsymbol{s}}=\underset{\boldsymbol{s}}{\arg \max } \bar{\lambda}(\boldsymbol{s}) .
$$

Using (41) we obtain

$$
\begin{aligned}
\hat{\boldsymbol{s}}=\underset{\boldsymbol{s}}{\arg \max } & \frac{1}{\sum_{k=1}^{K} N_{k}} \sum_{k=1}^{K} N_{k} \\
& \cdot\left[\frac{\boldsymbol{a}_{k}^{\mathrm{H}}(\boldsymbol{s}) \boldsymbol{C}_{k}^{-1}\left(\hat{\boldsymbol{R}}_{k}-\boldsymbol{C}_{k}\right) \boldsymbol{C}_{k}^{-1} \boldsymbol{a}_{k}(\boldsymbol{s})}{\left(\boldsymbol{a}_{k}^{\mathrm{H}}(\boldsymbol{s}) \boldsymbol{C}_{k}^{-1} \boldsymbol{a}_{k}(\boldsymbol{s})\right)^{2}}\right] .
\end{aligned}
$$

\footnotetext{
${ }^{7}$ The stopping condition at Step 4 can be tested either using a $\chi^{2}$ statistic or by comparing the level of the extracted point source to the noise level.

${ }^{8}$ The final MLE focusing operation can use the same update equations for an alternating coordinate maximization. In this case, we use the matrix inversion lemma twice: First we add the contribution of the last estimated coordinate to $C_{k}$, and then we subtract from $\boldsymbol{C}_{k}$ the estimated contribution of the coordinate along which we would like to optimize.
} 
TABLE III

ITERATIVE APPROXIMATE MAXIMUM LIKLIHOOD

1. Set the data covariance to be $\left\{\hat{\mathbf{R}}_{k}^{(0)}: k=1, \ldots, K\right\}$.

2. Set the noise covariance matrix to be $\mathbf{C}_{k}^{(0)}=\sigma^{2} \mathbf{I}$.

3. Let $\Delta_{k}^{(0)}=\hat{\mathbf{R}}_{k}^{(0)}-\mathbf{C}_{k}^{(0)}$.

4. Until the residual is noise like perform the following:

(a) Estimate the direction of a point source $\hat{\mathrm{s}}_{l}$ using (42).

(b) Estimate $\hat{\lambda}_{l}$ using (41).

(c) Let $\mathbf{C}_{k}^{(l+1)}=\mathbf{C}_{k}^{(l)}+\gamma \hat{\lambda}_{l} \mathbf{a}\left(\hat{\mathbf{s}}_{l}\right) \mathbf{a}\left(\hat{\mathbf{s}}_{l}\right)^{h}$

(d) Update $\Delta_{k}^{(l+1)}=\hat{\mathbf{R}}_{k}^{(0)}-\mathbf{C}_{k}^{(l+1)}$.

(e) Update $\left(\mathbf{C}_{k}^{(l+1)}\right)^{-1}$ using the matrix inversion rank 1 update formula.

(f) $l:=l+1$.

5. Use the estimated components to initialize a MLE search.

6. Reconstruct the image using the estimated directions, estimated powers and an ideal beam-shape.

From (7) we obtain that $C_{k}=\tilde{\boldsymbol{A}}_{k} \boldsymbol{B} \tilde{A}_{k}^{\mathrm{H}}+\sigma^{2} \boldsymbol{I}$, where $\tilde{\boldsymbol{A}}$ contains all the array responses toward the previously estimated astronomical sources. Assuming now that the power of the astronomical sources is negligible compared to the noise power (this is reasonable in many circumstances) and noting that $\|\boldsymbol{a}(\boldsymbol{s})\|^{2}=p$ for every $\boldsymbol{s}$ by our normalization, we obtain that $\boldsymbol{C}_{k} \approx \sigma^{2} \boldsymbol{I}$ and that (44) simplifies to (20). An iterative application of (20) is exactly the operation of the CLEAN beam removing technique.

\section{CONCLUSIONS}

In this paper we have presented a parametric approach to radio-astronomical image formation. We have used this approach to adapt some known spectral estimators to the astronomical image formation problem. We analyzed the effect of interference suppression on imaging and proposed the necessary changes to the imaging step in order to accommodate the spatial filtering preprocessing. A new AML algorithm for deconvolution has been presented, and the CLEAN algorithm was derived by approximating the ML power estimates. Finally, we have presented some simulated images demonstrating some of the ideas presented, and demonstrating the possible advantages in the parametric approach that leads to improved resolution.

The work shows that the design of a new radio telescope can (and probably should) use phased-array techniques to mitigate RFI, but the imaging software will have to be changed accordingly.

In this paper we have only analyzed LS based deconvolution. In extension of this work [19] we analyze the MEM image formation, in the context of adaptive interference suppression, and propose suitable changes to the imaging. The possible applications of this work spans beyond the field of radio astronomy. One possible example is ISAR imaging in the presence of strong interferers.

\section{APPENDIX A}

In this appendix we derive (37). To simplify the derivation we omit the explicit dependence on $\boldsymbol{s}$. Let

Hence

$$
\boldsymbol{R}_{k}=\boldsymbol{C}_{k}\left[\boldsymbol{I}+\lambda \boldsymbol{C}_{k}^{-1} \boldsymbol{a}_{k} \boldsymbol{a}_{k}^{\mathrm{H}}\right] .
$$

$$
\boldsymbol{R}_{k}^{-1}=\left[\boldsymbol{I}+\lambda \boldsymbol{C}_{k}^{-1} a_{k} \boldsymbol{a}_{k}^{\mathrm{H}}\right]^{-1} \boldsymbol{C}_{k}^{-1} .
$$

To compute $\left[\boldsymbol{I}+\lambda \boldsymbol{C}_{k}^{-1} \boldsymbol{a}_{k} \boldsymbol{a}_{k}^{\mathrm{H}}\right]^{-1}$, we use the matrix inversion lemma obtaining

$$
\left[\boldsymbol{I}+\lambda \boldsymbol{C}_{k}^{-1} \boldsymbol{a}_{k} \boldsymbol{a}_{k}^{\mathrm{H}}\right]^{-1}=\boldsymbol{I}-\frac{\lambda}{1+\lambda \boldsymbol{a}_{k}^{\mathrm{H}} \boldsymbol{C}_{k}^{-1} \boldsymbol{a}_{k}} \boldsymbol{C}_{k}^{-1} \boldsymbol{a}_{k} \boldsymbol{a}_{k}^{\mathrm{H}} .
$$

Using $\operatorname{tr}\left(\boldsymbol{a} \boldsymbol{b}^{\mathrm{H}}\right)=\boldsymbol{b}^{\mathrm{H}} \boldsymbol{a}$, we obtain

$$
\operatorname{tr}\left(\boldsymbol{R}_{k}^{-1} \hat{\boldsymbol{R}}_{k}\right)=\operatorname{tr}\left(\boldsymbol{C}_{k}^{-1} \hat{\boldsymbol{R}}_{k}\right)-\frac{\lambda \boldsymbol{a}_{k}^{\mathrm{H}} \boldsymbol{C}_{k}^{-1} \hat{\boldsymbol{R}}_{k} \boldsymbol{C}_{k}^{-1} \boldsymbol{a}_{k}}{1+\lambda \boldsymbol{a}_{k}^{\mathrm{H}} \boldsymbol{C}_{k}^{-1} \boldsymbol{a}_{k}} .
$$

Since $\operatorname{tr}\left(\boldsymbol{C}_{k}^{-1} \hat{\boldsymbol{R}}_{k}\right)$ is independent of the parameters it can be omitted from the maximization.

To evaluate $\log \left(\left|\boldsymbol{I}+\lambda \boldsymbol{C}_{k}^{-1} \boldsymbol{a}_{k} \boldsymbol{a}_{k}^{\mathrm{H}}\right|\right)$ note that the vector $\boldsymbol{C}_{k}^{-1} \boldsymbol{a}_{k}$ is an eigenvalue of the rank one matrix $\lambda \boldsymbol{C}_{k}^{-1} \boldsymbol{a}_{k} \boldsymbol{a}_{k}^{\mathrm{H}}$ with eigenvalue $\lambda \boldsymbol{a}_{k}^{\mathrm{H}} \boldsymbol{C}_{k}^{-1} a_{k}$. Therefore, it is an eigenvector of $\boldsymbol{I}+\lambda \boldsymbol{C}_{k}^{-1} \boldsymbol{a}_{k} \boldsymbol{a}_{k}^{\mathrm{H}}$ with eigenvalue $1+\lambda \boldsymbol{a}_{k}^{\mathrm{H}} \boldsymbol{C}_{k}^{-1} \boldsymbol{a}_{k}$. All the other eigenvalues of $\boldsymbol{I}+\lambda \boldsymbol{C}_{k}^{-1} \boldsymbol{a}_{k} \boldsymbol{a}_{k}^{\mathrm{H}}$ are 1 . Hence since the determinant is the product of the eigenvalues

$$
\left|\boldsymbol{I}+\lambda \boldsymbol{C}_{k}^{-1} \boldsymbol{a}_{k} \boldsymbol{a}_{k}^{\mathrm{H}}\right|=1+\lambda \boldsymbol{a}_{k}^{\mathrm{H}} \boldsymbol{C}_{k}^{-1} \boldsymbol{a}_{k} .
$$

Substituting into $\mathcal{L}$ we obtain (37).

\section{APPENDIX B}

In this appendix we present the gradient of the log-likelihood and the Fisher information matrix for the likelihood function given in (32) and (34). This gives a possibility of obtaining rapidly convergent ML estimation, given a good initialization. We will not give the derivation in detail as it is rather standard. Similar derivation for the perfectly calibrated array model case can be found, e.g., in [33]. 


$$
\begin{aligned}
\frac{\partial \operatorname{vec}\left(\boldsymbol{R}_{k}\right)}{\partial \boldsymbol{l}}= & {\left[\begin{array}{c}
\lambda_{1}\left(\left(\frac{\partial \boldsymbol{a}_{k}}{\partial l}\right)^{\mathrm{H}}\left(\boldsymbol{s}_{1}\right) \boldsymbol{\Gamma}_{k}^{\mathrm{H}} \otimes \boldsymbol{\Gamma}_{k} \boldsymbol{a}_{k}\left(\boldsymbol{s}_{1}\right)+\boldsymbol{a}_{k}^{\mathrm{H}}\left(\boldsymbol{s}_{1}\right) \boldsymbol{\Gamma}_{k}^{\mathrm{H}} \otimes \boldsymbol{\Gamma}_{k} \frac{\partial \boldsymbol{a}_{k}}{\partial l}\left(\boldsymbol{s}_{1}\right)\right)^{T} \\
\vdots \\
\lambda_{d}\left(\left(\frac{\partial \boldsymbol{a}_{k}}{\partial l}\right)^{\mathrm{H}}\left(\boldsymbol{s}_{d}\right) \boldsymbol{\Gamma}_{k}^{\mathrm{H}} \otimes \boldsymbol{\Gamma}_{k} \boldsymbol{a}_{k}\left(\boldsymbol{s}_{d}\right)+\boldsymbol{a}_{k}^{\mathrm{H}}\left(\boldsymbol{s}_{d}\right) \boldsymbol{\Gamma}_{k}^{\mathrm{H}} \otimes \boldsymbol{\Gamma}_{k} \frac{\partial \boldsymbol{a}_{k}}{\partial l}\left(\boldsymbol{s}_{d}\right)\right)^{T}
\end{array}\right]^{T} } \\
\frac{\partial \operatorname{vec}\left(\boldsymbol{R}_{k}\right)}{\partial \boldsymbol{m}}= & {\left[\begin{array}{c}
\lambda_{1}\left(\left(\frac{\partial \boldsymbol{a}_{k}}{\partial m}\right)^{\mathrm{H}}\left(\boldsymbol{s}_{1}\right) \boldsymbol{\Gamma}_{k}^{\mathrm{H}} \otimes \boldsymbol{\Gamma}_{k} \boldsymbol{a}_{k}\left(\boldsymbol{s}_{1}\right)+\boldsymbol{a}_{k}^{\mathrm{H}}\left(\boldsymbol{s}_{1}\right) \boldsymbol{\Gamma}_{k}^{\mathrm{H}} \otimes \boldsymbol{\Gamma}_{k} \frac{\partial \boldsymbol{a}_{k}}{\partial m}\left(\boldsymbol{s}_{1}\right)\right)^{T} \\
\vdots \\
\lambda_{d}\left(\left(\frac{\partial \boldsymbol{a}_{k}}{\partial m}\right)^{\mathrm{H}}\left(\boldsymbol{s}_{d}\right) \boldsymbol{\Gamma}_{k}^{\mathrm{H}} \otimes \boldsymbol{\Gamma}_{k} \boldsymbol{a}_{k}\left(\boldsymbol{s}_{d}\right)+\boldsymbol{a}_{k}^{\mathrm{H}}\left(\boldsymbol{s}_{d}\right) \boldsymbol{\Gamma}_{k}^{\mathrm{H}} \otimes \boldsymbol{\Gamma}_{k} \frac{\partial \boldsymbol{a}_{k}}{\partial m}\left(\boldsymbol{s}_{d}\right)\right)^{T}
\end{array}\right]^{T} }
\end{aligned}
$$

Let the parameter vector be

$$
\psi=\left[l, m, \lambda, \gamma_{1}, \cdots, \gamma_{K}\right]
$$

where $\boldsymbol{l}=\left[l_{1}, \cdots, l_{d}\right]$ is the vector of $l$ coordinate of the astronomical point sources, $\boldsymbol{m}=\left[m_{1}, \cdots, m_{d}\right]$ is the vector of $m$ coordinate of the astronomical point sources, and $\boldsymbol{s}_{i}=\left(l_{i}, m_{i}\right)$ are the location coordinates of the $i$ th astronomical source, $\lambda=$ $\left[\lambda_{1}, \cdots, \lambda_{d}\right]$ is the vector of brightness of the sources, and $\gamma_{k}$ are the calibration parameters at the $k$ th observation epoch. The score function is given by

$$
\frac{\partial \mathcal{L}}{\partial \psi}=-\sum_{k=1}^{K} N_{k} \frac{\partial \operatorname{vec}\left(\boldsymbol{R}_{k}\right)}{\partial \psi}\left(\boldsymbol{R}_{k}^{-T} \otimes \boldsymbol{R}_{k}^{-1}\right) \operatorname{vec}\left(\hat{\boldsymbol{R}}_{k}-\boldsymbol{R}_{k}\right) .
$$

and

$$
\boldsymbol{J}(\psi)=\sum_{k=1}^{K} N_{k}\left(\frac{\partial \operatorname{vec}\left(\boldsymbol{R}_{k}\right)}{\partial \psi}\right)^{\mathrm{H}}\left(\boldsymbol{R}_{k}^{-T} \otimes \boldsymbol{R}_{k}^{-1}\right) \frac{\partial \operatorname{vec}\left(\boldsymbol{R}_{k}\right)}{\partial \psi} .
$$

We now turn to compute $\frac{\partial \operatorname{vec}\left(\boldsymbol{R}_{k}\right)}{\partial \psi}$. Equation (32) can be rewritten as

$$
\begin{aligned}
& \operatorname{vec}\left(\boldsymbol{R}_{k}\right) \\
& \quad=\left[\boldsymbol{a}_{k}^{\mathrm{H}}\left(\boldsymbol{s}_{1}\right) \boldsymbol{\Gamma}_{k}^{\mathrm{H}} \otimes \boldsymbol{\Gamma}_{k} \boldsymbol{a}_{k}\left(\boldsymbol{s}_{1}\right), \cdots, \boldsymbol{a}_{k}^{\mathrm{H}}\left(\boldsymbol{s}_{d}\right) \boldsymbol{\Gamma}_{k}^{\mathrm{H}} \otimes \boldsymbol{\Gamma}_{k} \boldsymbol{a}_{k}\left(\boldsymbol{s}_{d}\right)\right] \boldsymbol{\lambda} .
\end{aligned}
$$

Hence we obtain

$$
\begin{aligned}
& \frac{\partial \operatorname{vec}\left(\boldsymbol{R}_{k}\right)}{\partial \boldsymbol{\lambda}} \\
& \quad=\left[\boldsymbol{a}_{k}^{\mathrm{H}}\left(\boldsymbol{s}_{1}\right) \boldsymbol{\Gamma}_{k}^{\mathrm{H}} \otimes \boldsymbol{\Gamma}_{k} \boldsymbol{a}_{k}\left(\boldsymbol{s}_{1}\right), \cdots, \boldsymbol{a}_{k}^{\mathrm{H}}\left(\boldsymbol{s}_{d}\right) \boldsymbol{\Gamma}_{k}^{\mathrm{H}} \otimes \boldsymbol{\Gamma}_{k} \boldsymbol{a}_{k}\left(\boldsymbol{s}_{d}\right)\right]
\end{aligned}
$$

and similarly we obtain (49) and (50) at the top of this page. Finally, the derivatives with respect to the calibration parameters are given by

$$
\begin{aligned}
\frac{\partial\left(\boldsymbol{R}_{k}\right)_{i j}}{\gamma_{n, k}}=\delta_{i, n} \sum_{l=1}^{d} a_{i}\left(\boldsymbol{s}_{l}\right) & \gamma_{j, k}^{*} a_{j}^{*}\left(\boldsymbol{s}_{l}\right) \\
& +\delta_{j, n} \sum_{l=1}^{d} \gamma_{i, k} a_{i}\left(\boldsymbol{s}_{l}\right) a_{j}^{*}\left(\boldsymbol{s}_{l}\right)
\end{aligned}
$$

\section{ACKNOWLEDGMENT}

The authors wish to thank E. Deprettere and their project partners at NFRA, especially A. van Ardenne, A. J. Boonstra, G. van Diepen, P. Friedman, A. Kokkeler, J. Noordam, and G. Schoonderbeek, for the very useful collaboration.

\section{REFERENCES}

[1] J. G. Ables, "Maximum entropy spectreal analysis," Astron. Astrophys. Supp., vol. 15, pp. 383-393, 1974.

[2] T. W. Anderson, An Introduction to Multivariate Statistics, ser. Wiley Publications in Statistics. New York: Wiley, 1958.

[3] C. Barnbaum and R. F. Bradley, "A new approach to interference excision in radio astronomy: Real time adaptive filtering," Astronom. J., vol. 115 , pp. $2598-2614,1998$.

[4] J. F. Böhme, "Estimation of spectral parameters of correlated signals in noise fields," Signal Processing, vol. 10, pp. 329-337, 1986.

[5] G. B. Borgiotti and L. J. Kaplan, "Supperresolution of uncorrelated interference sources by using adaptive array techniques," IEEE Trans. Antennas Propagat., vol. AP-27, pp. 842-845, 1979.

[6] J. Capon, "High resolution frequency-wavenumber spectrum analysis," Proc. IEEE, vol. 57, pp. 1408-1418, 1969.

[7] J. A. Fessler and A. O. Hero, "Space alternating generalized expectation maximization algorithm," IEEE Trans. Signal Processing, vol. 42, pp. 2664-2677, Oct. 1994.

[8] B. R. Frieden, "Restoring with maximum likelihood and maximum entropy," J. Opt. Soc. Amer., vol. 62, pp. 511-518, 1972.

[9] B. Friedlander and A. Zeira, "Eigenstructure based algorithms for direction finding with time varying arrays," IEEE Trans. Aerosp. Electron. Syst., vol. 32, pp. 689-700, Apr. 1996.

[10] P. Friedman, "A change point detection method for elimination of industrial interference in radio astronomy receivers," in Proc. 8th IEEE Signal Proc. Workshop on Statistical Signal Array Processing, 1996, pp. 264-266.

[11] S. F. Gull and G. J. Skilling, "Image reconstruction from incomplete and noisy data," Nature, vol. 272, pp. 686-690, 1978.

[12] J. A. Hogbom, "Aperture synthesis with nonregular distribution of interferometer baselines," Astron. Astrophys. Suppl., vol. 15, pp. 417-426, 1974.

[13] E. T. Jaynes, "Information theory and statistical mechanics," Phys. Rev., vol. 106, p. 620, 1957.

[14] — , "On the rational of maximum entropy methods," Proc. IEEE, vol. 70, pp. 939-952, 1982.

[15] B. L. Kasper, F. S. Chute, and D. Routledge, "Excising terrestrial radio interference in low frequency radio astronomy," Monthly Notices Roy. Astron. Soc., vol. 199, pp. 345-354, 1982.

[16] H. Krim and M. Viberg, "Sensor array signal processing: The parameter estimation approach," IEEE Signal Processing Mag., pp. 67-94, July 1996. 
[17] A. Leshem and A. J. van der Veen, "Detection and filtering in radioastronomy," Delft Univ. Technol., Delft, The Netherlands, Tech. Rep. NOEMI-98-01, 1998.

[18] _ "The effect of blanking of TDMA signals on radio-astronomical correlation measurements," in Proc. IEEE Workshop on Higher Order Statistics, Ceasaria, Israel, June 1999, pp. 25-29.

[19] — The Effect of Interference Mitigation Techniques on MEM Image Formation, 2000.

[20] A. Leshem, A. J. van der Veen, and A. J. Boonstra, "Multichannel interference mitigation techniques in radio astronomy," Astrophys. J. Suppl., Oct. 2000.

[21] A. Leshem, A. J. van der Veen, and E. Deprettere, "Detection and blanking of GSM signals in radio-astronomical observations," in Proc. IEEE SPAWC'99, May 1999.

[22] S. Maslakovic, I. R. Linscott, M. Oslick, and J. D. Twicken, "Excising radio frequency interference using the discrete wavelet transform," in Proc. IEEE Signal Processing Int. Symp. Time-Frequency and TimeScale Analysis, New York, 1996, pp. 349-352.

[23] J. E. Noordam and A. G. de Bruyn, "High dynamic range mapping of radio sources, with application to 3C84," Nature, vol. 299, pp. 597-600, 1982.

[24] J. A. O'Sullivan, R. E. Blahut, and D. L. Snyder, "Information theoretic image formation," IEEE Trans. Inform. Theory, vol. 44, pp. 2094-2123, Oct. 1998.

[25] T. J. Pearson and A. C. S. Readhead, "Image formation by self-calibration in radio astronomy," Аnnu. Rev. Astron. Astrophys., vol. 22, pp. 97-130, 1984

[26] R. A. Perley, F. Schwab, and A. H. Bridle, Eds., Synthesis Imaging in Radio Astronomy. San Francisco, CA: Astron. Soc. Pacific, 1989

[27] J. A. Roberts, Ed., Indirect Imaging. Cambridge, U.K.: Cambridge Univ. Press, 1984

[28] K. Rohlfs and T. L. Wilson, Tools of Radio Astronomy, 3rd ed, ser. A \& A Library. New York: Springer-Verlag, 1999.

[29] M. Ryle, "A new radio interferometer and its application to the observation of weak stars," Proc. Roy. Soc. A, vol. 211, pp. 351-375, 1952

[30] _ "The new Cambridge radio telescope," Nature, vol. 194, pp. $517-518,1962$

[31] U. J. Schwarz, "Mathematical-statistical description of the iterative beam removing technique (method CLEAN)," Astron. Astrophys., vol. 65 , pp. 345-356, 1978 .

[32] — "The method CLEAN_-Use missuse and variations," in Image Formation From Coherence Functions in Astronomy, C. van Schoonveld, Ed. Dordrecht, The Netherlands: Reidel, 1979, pp. 261-275.
[33] J. Sheinvald, "On detection and localization of multiple signals by sensor arrays," Ph.D. dissertation, Tel-Aviv Univ., Tel-Aviv, Israel, 1998.

[34] J. Sheinvald, M. Wax, and A. J. Weiss, "Localization of multiple sources using moving arrays," IEEE Trans. Signal Processing, vol. 46, pp. 2736-2743, Oct. 1998.

[35] D. L. Snyder, J. A. O'Sullivan, and M. I. Miller, "The use of maximum likelihood estimation for forming imaging of diffuse radar targets from delay-doppler data," IEEE Trans. Inform. Theory, vol. 35, pp. 536-548, May 1989.

[36] P. Stoica and A. Nehorai, "MUSIC, maximum likelihood, and Cramer-Rao bound," IEEE Trans. Acoust., Speech, Signal Processing, vol. 37, pp. 720-743, May 1989

[37] S. M. Tan, "An analysis of the properties of CLEAN and smoothness stabilized CLEAN-Some warnings," Month. Not. Roy. Astron. Soc., vol. 220, pp. 971-1001, 1986.

[38] A. R. Thompson, "The response of radio-astronomy synthesis array to interfering signals," IEEE Trans. Antennas Propagat., vol. AP-30, pp 450-456, May 1982.

[39] A. R. Thompson, J. M. Moran, and G. W. Swenson, Interferometry and Synthesis in Radioastronomy. New York: Wiley, 1986.

[40] K. Sharman, T. Durani, M. Wax, and T. Kailath, "Asymptotic performance of eigenstructure spectral analysis methods," in Proc. IEEE Int Conf. Acoust., Speech, Signal Processing 1984, San Diego, CA, Mar. 1996, pp. 4.5.51-4.5.54.

[41] M. Wax and A. Leshem, "Joint estimation of directions-of-arrival and time-delays of multiple reflections of a known signal," IEEE Trans. Signal Processing, vol. 45, no. 10, pp. 2477-2484, Oct. 1997.

[42] R. Weber, C. Faye, F. Biraud, and J. Dansou, "Spectral detector for interference time blanking using quantized correlator," Astron. Astrophys. Suppl., vol. 126, no. 1, pp. 161-167, Nov. 1997.

[43] S. J. Wernecke, "Two dimensional maximum entropy reconstruction of radio brightness," Radio Sci., vol. 12, pp. 831-844, 1977.

[44] J. L. Yen, "Image reconstruction in synthesis radio telescopes," in Array Signal Processing. ser. Signal Processing, S. Haykin, Ed. Englewood Cliffs, NJ: Prentice-Hall, 1985.

[45] A. Zeira and B. Friedlander, "Direction finding with time varying arrays," IEEE Trans. Signal Processing, vol. 43, pp. 927-937, Apr. 1995.

[46] I. Ziskind and M. Wax, "Maximum likelihood localization of multiple sources by alternating projections," IEEE Trans. Acoust., Speech, Signal Processing, vol. 36, pp. 1553-1560, Oct. 1988 